\title{
1 Polyhedral Dynamics and the Jahn-Teller Effect
}

\author{
R. BRUCE KING \\ Department of Chemistry, University of Georgia, Athens, GA 30602, USA
}

\section{INTRODUCTION}

The role of polyhedra in the static description of chemical structures makes of considerable interest their dynamic properties. In this connection the central concept in the study of polyhedral dynamics is that of a polyhedral isomerization. In this context a polyhedral isomerization is defined as a deformation of a specific polyhedron $\mathcal{P}_{1}$ until its vertices define a new polyhedron $\mathcal{P}_{2}$. Of particular interest are sequences of two polyhedral isomerization steps $\mathcal{P}_{1} \rightarrow \mathcal{P}_{2} \rightarrow \mathcal{P}_{3}$ in which the final polyhedron $\mathcal{P}_{3}$ is combinatorially equivalent to the initial polyhedron $\mathcal{P}_{1}$ although with some permutation of the vertices. Such polyhedral isomerization sequences are called degenerate polyhedral isomerizations.

Polyhedral isomerizations may be studied using either a microscopic or macroscopic approach. The microscopic approach uses details of polyhedral topology to elucidate possible single polyhedral isomerization steps, namely which types of isomerization steps are possible. Such isomerization steps consist most commonly of so-called diamond-square-diamond processes or portions thereof. The microscopic approach to polyhedral isomerizations is relevant to understanding fluxional processes in borane and metallaborane polyhedra.

The earliest work on polyhedral isomerizations by Muetterties [1-4], Gielen [5-12], Musher [13,14], Klemperer [15-17] and Brocas [18,19] used a macroscopic approach involving relationships between different permutational isomers. Such relationships may be depicted by reaction graphs called topological representations (or top-reps) in which the vertices correspond to different permutational isomers and the edges correspond to single degenerate polyhedral isomerization steps. The macroscopic point of view is also useful for the classification of Jahn-Teller distortions of polyhedra [20] relating to the instability of a non-linear symmetrical nuclear configuration in an electronic degenerate state [21-24]. 
Both approaches will be reviewed in this chapter, which expands and updates reviews published by the author in 1988 [25] and 1994 [26] and a book in 1992 [27].

\section{POLYHEDRON TOPOLOGY}

Before considering polyhedral dynamics it is first necessary to consider the static topology of polyhedra. Of fundamental importance are relationships between possible numbers and types of vertices $(v)$, edges $(e)$, and faces $(f)$ of polyhedra. In this connection the following elementary relationships are particularly significant [28]:

1. Euler's relationship:

$$
v-e+f=2
$$

This theorem is illustrated by the five regular polyhedra depicted in Figure 1.1.

2. Relationship between the edges and faces:

$$
\sum_{i=3}^{v-1} i f_{i}=2 e
$$

In Equation (1.2) $f_{i}$ is the number of faces with $i$ edges (i.e., $f_{3}$ is the number of triangular faces, $f_{4}$ is the number of quadrilateral faces, etc.). This relationship arises from the fact that exactly two faces share each edge of the polyhedron. Since no face can have fewer edges than the three of a triangle, the following inequality must hold in all cases:

$$
3 f \leq 2 e
$$

3. Relationship between the edges and vertices:

$$
\sum_{i=3}^{v-1} i v_{i}=2 e
$$

In Equation (1.4) $v_{i}$ is the number of vertices of degree $i$ (i.e., having $i$ edges meeting at the vertex in question). This relationship arises from the fact that each edge of the polyhedron connects exactly two vertices. Since no vertex of a polyhedron can have a degree less than three, the following inequality must hold in all cases:

$$
3 v \leq 2 e
$$

4. Totality of faces:

$$
\sum_{i=3}^{v-1} f_{i}=f
$$




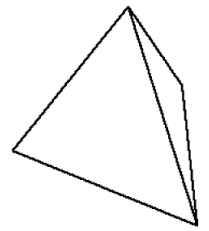

Tetrahedron

$v=4, e=6, f=4$

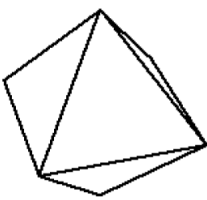

Octahedron

$v=6, e=12, f=8$

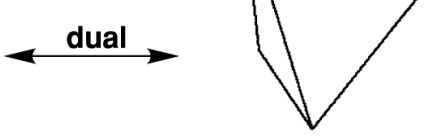

Tetrahedron

$v=4, e=6, f=4$

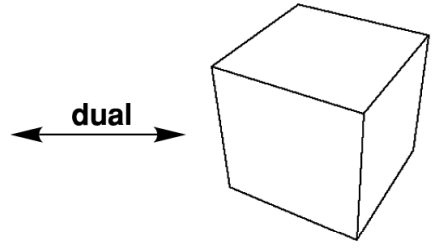

Cube

$v=8, e=12, f=6$
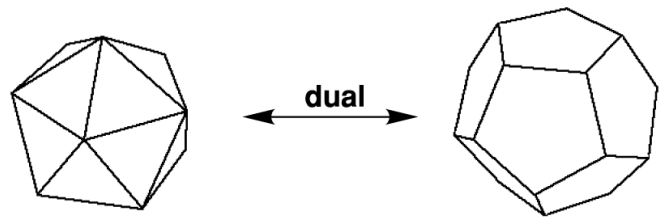

Dodecahedron

Icosahedron

$v=12 . e=30, f=20$ $v=20, e=30, f=12$

Figure 1.1 The five regular polyhedra depicted as dual pairs.

5. Totality of vertices:

$$
\sum_{i=3}^{v-1} v_{i}=v
$$

Equation (1.6) relates the $f_{i}$ s to $f$ and Equation (1.7) relates the $v_{i}$ s to $v$.

In generating actual polyhedra, the operations of capping and dualization are often important. Capping a polyhedron $\mathcal{P}_{1}$ consists of adding a new vertex above the center of one of its faces $\mathcal{F}_{1}$ followed by adding edges to connect the new vertex with each vertex of $\mathcal{F}_{1}$. If $\mathcal{F}_{1}$ has $k$ edges, then the capping process can be more specifically described as $k$-capping. 


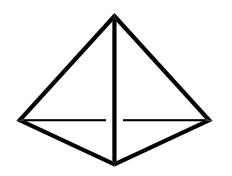

Tetrahedron

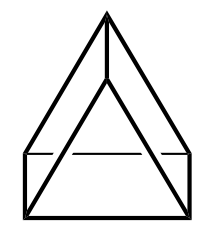

Trigonal Prism
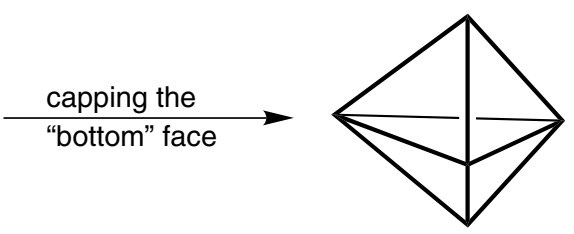

Trigonal Bipyramid

capping the 3

rectangular

faces

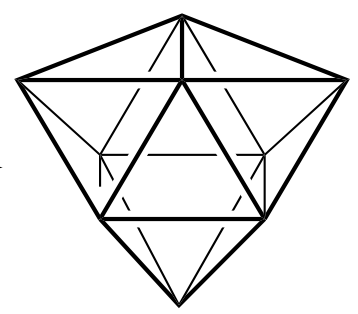

4,4,4-Tricapped Trigonal Prism

Figure 1.2 (a) Capping a face of the tetrahedron to form a trigonal bipyramid. (b) Capping all three rectangular faces of a trigonal prism to form a $D_{3 h}$ tricapped trigonal prism.

The capping process gives a new polyhedron $\mathcal{P}_{2}$ having one more vertex than $\mathcal{P}_{1}$. If a triangular face is capped (i.e., 3-capping), the following relationships will be satisfied in which the subscripts 1 and 2 refer to $\mathcal{P}_{1}$ and $\mathcal{P}_{2}$, respectively:

$$
v_{2}=v_{1}+1 ; \quad e_{2}=e_{1}+3 ; \quad f_{2}=f_{1}+2
$$

Such a capping of a triangular face is found in the capping of a tetrahedron to form a trigonal bipyramid (Figure 1.2a). In general if a face with $f_{k}$ edges is capped (i.e., $k$-capping), the following relationships will be satisfied:

$$
v_{2}=v_{1}+1 ; \quad e_{2}=e_{1}+f_{k} ; \quad f_{2}=f_{1}+f_{k}-1
$$

For example, application of such a capping process to each of the three rectangular faces of a trigonal prism generates the 4,4,4-tricapped trigonal prism (Figure 1.2b). This nine-vertex deltahedron appears in both coordination chemistry (e.g., $\mathrm{ReH}_{9}{ }^{2-}$ ) and borane chemistry (e.g., $\mathrm{B}_{9} \mathrm{H}_{9}{ }^{2-}$ ).

Another process of interest in polyhedral topology is the dualization of polyhedra. A given polyhedron $\mathcal{P}$ can be converted into its dual $\mathcal{P}^{*}$ by locating the centers of the faces of $\mathcal{P}^{*}$ at the vertices of $\mathcal{P}$ and the vertices of $\mathcal{P}^{*}$ above the centers of the faces of $\mathcal{P}$. Two vertices in the dual $\mathcal{P}^{*}$ are connected by an edge when the corresponding faces in $\mathcal{P}$ share an edge. 
The process of dualization has the following properties:

(1) The numbers of vertices and edges in a pair of dual polyhedra $\mathcal{P}$ and $\mathcal{P}^{*}$ satisfy the relationships $v^{*}=f, e^{*}=e, f^{*}=v$, in which the starred variables refer to the dual polyhedron $\mathcal{P}^{*}$. Thus for the octahedron $(\mathcal{P}) / \operatorname{cube}\left(\mathcal{P}^{*}\right)$ dual pair depicted in Figure $1.1 v=f^{*}=6, e^{*}=e=12$, $f=v^{*}=8$.

(2) Dual polyhedra have the same symmetry elements and thus belong to the same symmetry point group. Thus in the example above both the octahedron and the cube have the $O_{h}$ symmetry point group. Also note in general that the dualization of a prism gives the corresponding bipyramid and vice versa.

(3) Dualization of the dual of the polyhedron leads to the original polyhedron.

(4) The degrees of the vertices of a polyhedron correspond to the number of edges in the corresponding face polygons in its dual.

The process of dualization can be illustrated by the regular polyhedra (Figure 1.1). Thus the octahedron and cube are dual to each other as are the icosahedron and dodecahedron. The tetrahedron is self-dual.

The problem of the classification and enumeration of polyhedra is a complicated one. Thus there appear to be no formulas, direct or recursive, for which the number of combinatorially distinct polyhedra having a given number of vertices, edges, faces, or any given combination of these elements can be calculated $[29,30]$. Duijvestijn and Federico have enumerated by computer the polyhedra having up to 22 edges according to the numbers of vertices, edges, and faces and their symmetry groups and present a summary of their methods, results, and literature references to previous work [31]. Their work shows that there are $1,2,7,34,257,2606$, and 32,300 topologically distinct polyhedra having 4 , $5,6,7,8,9$, and 10 faces or vertices, respectively. Tabulations are available for all $301(=1+2+7+34+257)$ topologically distinct polyhedra having eight or fewer faces [32] or eight or fewer vertices [33]. These two tabulations are essentially equivalent by the dualization relationship discussed above.

In coordination chemistry, the polyhedra of greatest significance in coordination chemistry are those that can be formed by the nine orbitals of the $\mathrm{sp}^{3} \mathrm{~d}^{5}$ valence orbital manifold accessible to d-block transition metals. There are, however, some polyhedra having fewer than nine vertices that cannot be formed by these nine orbitals; such polyhedra are called forbidden polyhedra $[34,35]$. Group theoretical arguments show that polyhedra of the following types are always forbidden polyhedra:

(1) Polyhedra having eight vertices, a direct product symmetry group $R \times C_{S}$ or $R \times C_{i}$ ( $R$ contains only proper rotations) and the plane in $C_{s}$ fixing either 0 or 6 vertices.

(2) Polyhedra having a six-fold or higher $C_{n}$ rotation axis. 


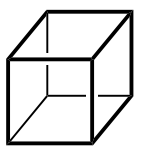

Cube $\left(O_{h}\right)$

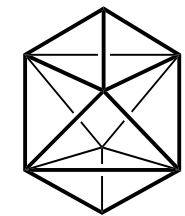

Bicapped octahedron $\left(D_{3 d}\right)$

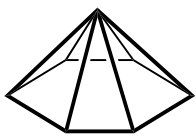

Hexagonal pyramid $\left(C_{6 v}\right)$

Figure 1.3 Chemically significant forbidden polyhedra: (a) the hexagonal pyramid; (b) examples of eight-vertex polyhedra (all with inversion centers) which cannot be formed from a nine-orbital $\mathrm{sp}^{3} \mathrm{~d}^{5}$ manifold.

Chemically significant forbidden polyhedra include the seven-vertex hexagonal bipyramid and the eight-vertex cube, $D_{3 d}$ bicapped octahedron, $D_{3 h} 3,3-$ bicapped trigonal prism, and hexagonal bipyramid (Figure 1.3).

In the chemistry of polyhedral boranes and related compounds including their organometallic derivatives, the polyhedra of greatest importance are deltahedra, i.e., polyhedra in which all faces are triangles. Among the large number of such possible deltahedra, the most important deltahedra are the so-called 'most spherical' deltahedra, i.e., those where the vertex degrees are as similar as possible to each other. In terms of the structures of the deltahedral boranes $\mathrm{B}_{n} \mathrm{H}_{n}{ }^{2-}$, this means deltahedra having from six to 12 vertices in which all of the vertices are of degree 4 or degree 5 (Figure 1.4).

\section{POLYHEDRAL ISOMERIZATIONS: MICROSCOPIC MODELS}

\subsection{DIAMOND-SQUARE-DIAMOND PROCESSES}

A polyhedral isomerization can be defined as a deformation of a specific polyhedron $\mathcal{P}_{1}$ until its vertices define a new polyhedron $\mathcal{P}_{2}$. Of particular interest are sequences of two polyhedral isomerization steps $\mathcal{P}_{1} \rightarrow \mathcal{P}_{2} \rightarrow \mathcal{P}_{3}$ in which the final polyhedron $\mathcal{P}_{3}$ is combinatorially (i.e., topologically) equivalent to the initial polyhedron $\mathcal{P}_{1}$ although with some permutation of its vertices, generally not the identity permutation. In this sense two polyhedra $\mathcal{P}_{1}$ and $\mathcal{P}_{3}$ may be considered to be combinatorially equivalent [36] when there are three one-to-one mappings $\mathcal{V}, \mathcal{E}$, and $\mathcal{F}$ from the vertex, edge, and face sets, respectively, of $\mathcal{P}_{1}$ to 


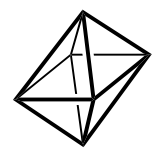

6 vertices: Octahedron

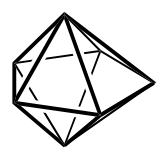

7 vertices:

Pentagonal

Bipyramid

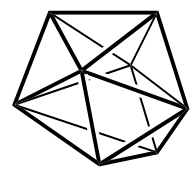

8 vertices:

Bisdisphenoid

("D2d Dodecahedron")

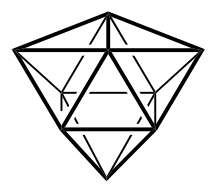

9 vertices:

4,4,4-Tricapped Trigonal Prism

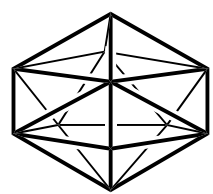

11 vertices:

Edge-coalesced Icosahedron

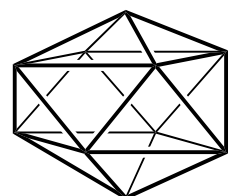

10 vertices:

4,4-Bicapped

Square Antiprism

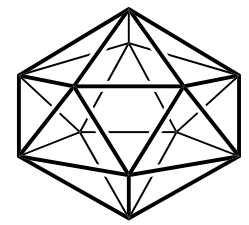

12 vertices: Icosahedron

Figure 1.4 The deltahedra found in the boranes $\mathrm{B}_{n} \mathrm{H}_{n}{ }^{2-}$ and isoelectronic carboranes $(6 \leq n \leq 12)$.

the corresponding sets of $\mathcal{P}_{3}$ such that incidence relations are preserved. Thus if a vertex, edge, or face $\alpha$ of $\mathcal{P}_{1}$ is incident to or touches upon a vertex, edge, or face $\beta$ of $\mathcal{P}_{1}$, then the images of $\alpha$ and $\beta$ under $\mathcal{V}, \mathcal{E}$, or $\mathcal{F}$ are incident in $\mathcal{P}_{3}$.

Consider a polyhedral isomerization sequence $\mathcal{P}_{1} \rightarrow \mathcal{P}_{2} \rightarrow \mathcal{P}_{3}$ in which $\mathcal{P}_{1}$ and $\mathcal{P}_{3}$ are combinatorially equivalent. Such a polyhedral isomerization sequence may be called a degenerate polyhedral isomerization with $\mathcal{P}_{2}$ as the intermediate polyhedron. Structures undergoing such degenerate polyhedral isomerization processes are often called fluxional [37]. A degenerate polyhedral isomerization with a planar intermediate 'polyhedron' (actually a polygon) may be called a planar polyhedral isomerization. The simplest example of a planar polyhedral isomerization is the interconversion of two enantiomeric tetrahedra 


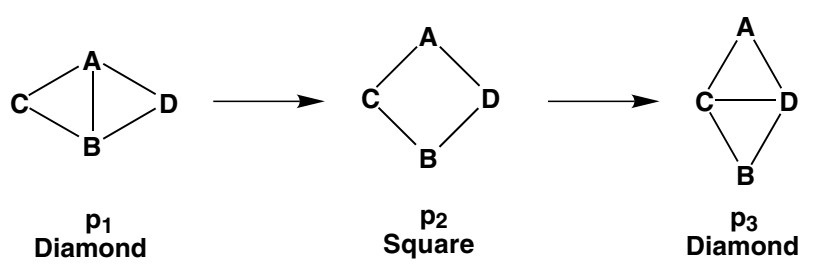

Figure 1.5 The effect of a diamond-square-diamond (dsd) process on a pair of triangular faces sharing an edge.

$\left(\mathcal{P}_{1}\right.$ and $\left.\mathcal{P}_{3}\right)$ through a square planar intermediate $\mathcal{P}_{2}$. Except for this simplest example, planar polyhedral isomerizations are unfavorable owing to excessive intervertex repulsion.

Microscopic approaches to polyhedral isomerizations dissect such processes into elementary steps. The most important elementary step is the diamondsquare-diamond process that was first recognized in a chemical context by Lipscomb in 1966 [38] as a generalization of a process proposed earlier by Berry [39] for the degenerate isomerization of a trigonal bipyramid. Such a diamond-square-diamond process or 'dsd process' in a polyhedron occurs at two triangular faces sharing an edge (Figure 1.5). Thus a configuration such as $\mathrm{p}_{1}$ can be called a dsd situation and the edge $\mathrm{AB}$ can be called a switching edge.

Let $a, b, c$, and $d$ represent the degrees of the vertices $\mathrm{A}, \mathrm{B}, \mathrm{C}$, and $\mathrm{D}$, respectively, in $\mathrm{p}_{1}$, then the dsd type of the switching edge $\mathrm{AB}$ can be represented as $a b(c d)$. In this designation the first two digits refer to the degrees of the vertices joined by $A B$ but contained in the faces (triangles) having $A B$ as the common edge (i.e., $C$ and $D$ in $p_{1}$ ). The quadrilateral face formed in structure $p_{2}$ may be called a pivot face. If $a, b, c$, and $d$ are the degrees of vertices A, B, C, and D, respectively, in the original diamond $\left(p_{1}\right)$, then the requirement for a degenerate dsd process is the following:

$$
c=a-1 \text { and } d=b-1 \quad \text { or } c=b-1 \text { and } d=a-1
$$

A polyhedron with $e$ edges has $e$ distinct dsd situations; if at least one of these dsd situations is degenerate by satisfying Equation (1.10), then the polyhedron is inherently fluxional [40]. The inherent rigidity/fluxionality of the most important deltahedra including the most spherical deltahedra found in boranes (Figure 1.4) is summarized in Table 1.1.

This simple analysis indicates that in deltahedral structures the 4, 6, 10, and 12 vertex structures are inherently rigid; the 5, 8, 9, and 11 vertex structures are inherently fluxional; and the rigidity of the seven-vertex structure depends upon the energy difference between the two most symmetrical seven-vertex deltahedra [40]. From the point of view of coordination chemistry, note that the trigonal bipyramid and bisdisphenoid, which are favored coordination polyhedra for 
Table 1.1 Inherent fluxionality/rigidity of deltahedra

\begin{tabular}{cll}
\hline Vertices & Deltahedron & Inherently rigid or fluxional \\
\hline 4 & Tetrahedron & Rigid \\
5 & Trigonal bipyramid & Fluxional \\
6 & Octahedron & Rigid \\
7 & Pentagonal bipyramid/ & ML $_{7} \rightarrow$ Fluxional \\
& Capped octahedron & Boranes $\rightarrow$ Rigid \\
8 & Bisdisphenoid & Fluxional \\
9 & Tricapped trigonal prism & Fluxional \\
10 & Bicapped square antiprism & Rigid \\
11 & Edge-coalesced icosahedron & Fluxional \\
12 & Icosahedron & Rigid \\
\hline
\end{tabular}

coordination numbers 5 and 8, respectively, are inherently fluxional whereas the octahedron found in six-coordinate complexes is inherently rigid. This relates to the stereochemical non-rigidity of five- and eight-coordinate complexes observed experimentally in contrast to the relative rigidity of six-coordinate complexes. In the case of deltahedral borane chemistry, experimental fluxionality observations by boron-11 nuclear magnetic resonance on $\mathrm{B}_{n} \mathrm{H}_{n}{ }^{2-}(6 \leq n \leq 12)$ indicate the 6-, 7-, 9-, 10-, and 12-vertex structures to be rigid and the 8- and 11 -vertex structures to be fluxional. The only discrepancy between such experiments and these very simple topological criteria for fluxionality in the deltahedral boranes arises in the nine-vertex structure $\mathrm{B}_{9} \mathrm{H}_{9}{ }^{2-}$.

The discrepancy between the predictions of this simple topological approach and experiment for $\mathrm{B}_{9} \mathrm{H}_{9}{ }^{2-}$ has led to the search for more detailed criteria for the rigidity of deltahedral boranes. In this connection Gimarc and Ott have studied orbital symmetry methods, particularly for the five-[41], seven-[42], and nine-[43] vertex borane and carborane structures. A topologically feasible dsd process is orbitally forbidden if crossing of occupied and vacant molecular orbitals (i.e., a 'HOMO-LUMO crossing') occurs during the dsd process as illustrated in Figure 1.6 for the single dsd process for the trigonal bipyramid, namely the so-called Berry pseudorotation $[39,44]$. For such an orbitally forbidden process the activation barrier separating initial and final structures is likely to be large enough to prevent this polyhedral isomerization. However, the forbidden dsd polyhedral rearrangement for the five-vertex $\mathrm{B}_{5} \mathrm{H}_{5}{ }^{2-}$ and corresponding carboranes is allowed in $\mathrm{ML}_{5}$ coordination compounds and has been observed in $\mathrm{PX}_{5}$ derivatives such as $\mathrm{PC}_{5}$ and $\mathrm{PF}_{5}$ (i.e., the single fluorine-19 resonance in $\mathrm{PF}_{5}$ ) as well as $\mathrm{Fe}(\mathrm{CO})_{5}$ (i.e., the single carbon-13 or oxygen-17 resonance). Guggenberger and Muetterties [45] point out that cage framework rearrangements such as those in the deltahedral boranes and carboranes involve bond stretches which must require more energy than bond angle changes that occur in coordination polyhedra of ligands bound to a central atom. 

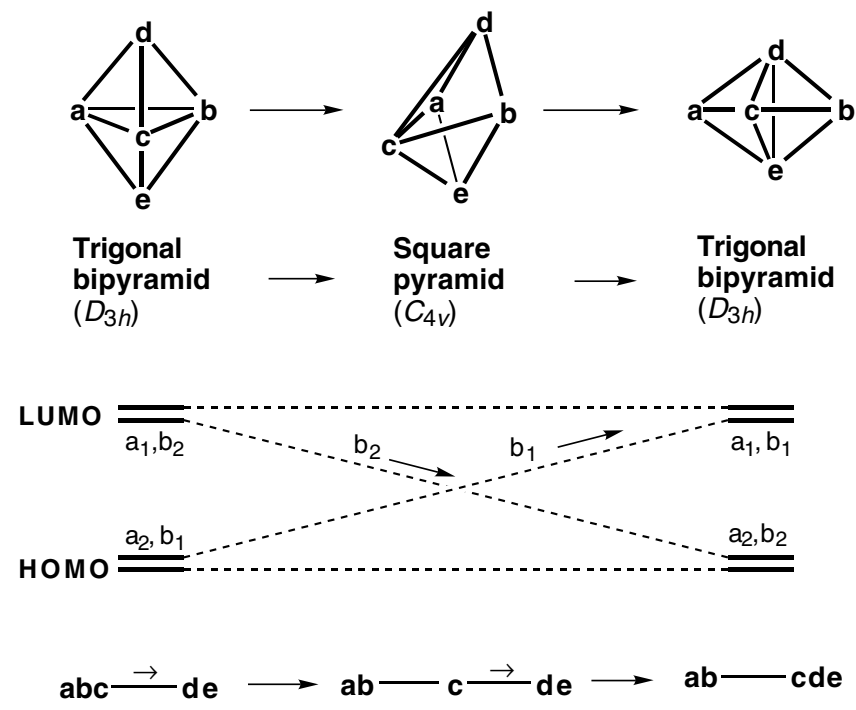

Figure 1.6 (a) The single dsd process converting a trigonal bipyramid to an isomeric trigonal bipyramid through a square pyramid intermediate (Berry pseudorotation). (b) The orbital crossing for this dsd process. (c) Gale diagrams for this polyhedral isomerization sequence.

Some selection rules have been proposed for distinguishing between symmetry-allowed and symmetry-forbidden processes in deltahedral boranes, carboranes, and related structures. Thus Wales and Stone [46] distinguish between symmetry-allowed and symmetry-forbidden processes by observing that a HOMO-LUMO crossing occurs if the proposed transition state has a single atom lying on a principal $C_{n}$ rotational axis where $n \geq 3$. A more detailed selection rule was observed by Mingos and Johnston [47]. If the four outer edges of the two fused triangular faces (i.e., the 'diamond') are symmetry equivalent, then a single dsd process results in a pseudorotation of the initial polyhedron by $90^{\circ}$ (Figure 1.7a) such as occurs for the trigonal bipyramid in Figure 1.6. However, if the edges are not symmetry equivalent (as indicated by the regular and bold edges in Figure 1.7b), then the rearrangement results in a pseudoreflection of the initial polyhedron. Pseudorotations are symmetry forbidden and have larger activation energies than pseudoreflections, which are symmetry allowed. Subsequent work by Wales et al. [48] has shown that a dsd process in which a mirror plane is retained throughout involves an orbital crossing and is therefore symmetry forbidden in accord with the earlier work by Gimarc and Ott [41].

The fact that a degenerate single dsd isomerization of the $D_{3 h}$ nine-vertex tricapped trigonal prismatic $\mathrm{B}_{9} \mathrm{H}_{9}{ }^{2-}$ through a $C_{4 v}$ capped square antiprismatic intermediate (Figure 1.8a) is orbitally forbidden [43] has stimulated searches 


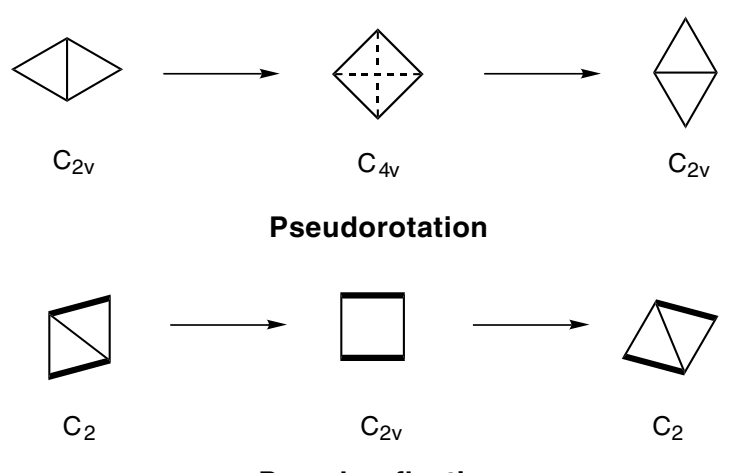

Figure 1.7 (a) A pseudorotation process involving an intermediate with a $C_{4}$ axis. (b) A pseudoreflection process involving an intermediate without a $C_{4}$ axis.

for other isomerization mechanisms for the tricapped trigonal prism. In this connection Gimarc and Ott [43] have found a double dsd isomerization of $\mathrm{B}_{9} \mathrm{H}_{9}{ }^{2-}$ (Figure 1.8b) to be orbitally allowed. However, Ceulemans et al. [49] have shown by computation that an analogous double dsd isomerization of the carborane $\mathrm{C}_{2} \mathrm{~B}_{7} \mathrm{H}_{9}$ passes through an intermediate with an open hexagonal face thereby preserving $C_{3 v}$ subgroup symmetry of the $D_{3 h}$ point group of the original tricapped trigonal prism.

\subsection{GALE DIAGRAMS}

Gale diagrams provide an elegant mathematical method for the study of microscopic aspects of rearrangement of polyhedra having relatively few vertices (i.e., for $v \leq 6$ ) by reducing the dimensionality of the allowed vertex motions. In a chemical context Gale diagrams can be used to study possible rearrangements of six-atom structures. Using this approach the skeletal rearrangements of the six atoms are depicted as movements of six points on the circumference of a circle or from the circumference to the center of the circle subject to severe restrictions that reduce possible such movements to a manageable number [50].

Consider a polytope $\mathcal{P}$ in $d$-dimensional space $\mathfrak{R}^{d}$, where the term 'polytope' refers to the generalization of the concept of 'polyhedron' to any number of dimensions [36]. The minimum number of vertices of such a polytope is $d+1$ and there is only one such polytope, namely the $d$-simplex in which each possible pair of the $d+1$ vertices are connected by an edge corresponding to the so-called complete graph [51] $K_{d+1}$. The combinatorially distinct possibilities for $d$-dimensional polytopes having only $d+2$ and $d+3$ vertices (polyhedra with 'few' vertices) are also rather limited. They can be represented faithfully in a space of less than $d$ dimensions through a Gale 

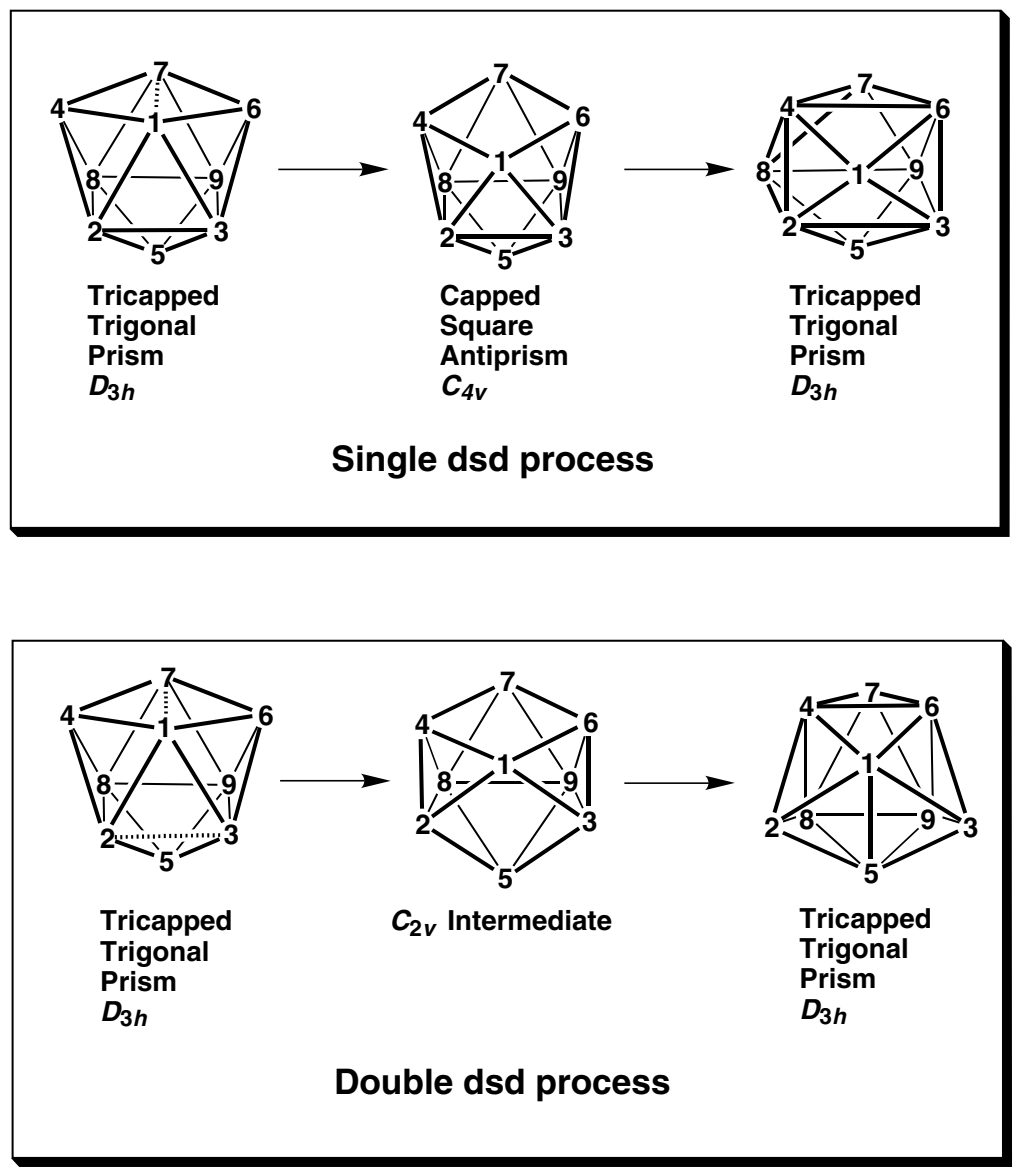

Figure 1.8 (a) A forbidden single dsd degenerate isomerization of a tricapped trigonal prism. (b) An allowed double dsd degenerate isomerization of a tricapped trigonal prism.

transformation [52]. More specifically, if $\mathcal{P}$ is a $d$-dimensional polytope with $v$ vertices, a Gale transformation leads to a Gale diagram of $\mathcal{P}$ consisting of $v$ points in $(v-d-1)$-dimensional space $\Re^{d-1}$ in one-to-one correspondence with the vertices of $\mathcal{P}$. From the Gale diagram it is possible to determine all of the combinatorial properties of $\mathcal{P}$ such as the subsets of the vertices of $\mathcal{P}$ that define faces of $\mathcal{P}$, the combinatorial types of these faces, etc. The combinatorial properties of a polytope $\mathcal{P}$ which can be determined by the Gale diagram include all possible isomerizations (rearrangements) of $\mathcal{P}$ to other polytopes having the same number of vertices and imbedded in the same number of dimensions as $\mathcal{P}$. Also of particular importance is the fact that, if $v$ is not much larger than $d$ (i.e., if $v \leq 2 d$ ), then the dimension of the Gale diagram is smaller than that of the original polytope $\mathcal{P}$. 
Now consider polyhedra in the ordinary three-dimensional space of interest in chemical structures (i.e., $d=3$ ). Gale diagrams of five- and six-vertex polyhedra can be embedded into one- or two-dimensional space, respectively, thereby simplifying analysis of their possible vertex motions leading to nonplanar polyhedral isomerizations of these polyhedra of possible interest in a chemical context.

In order to obtain a Gale diagram for a given polyhedron, the polyhedron is first subjected to a Gale transformation. Consider a polyhedron with $v$ vertices as a set of $v$ points $X_{1}, \ldots, X_{v}$ in three-dimensional space $\mathfrak{R}^{3}$. These points may be regarded as three-dimensional vectors $\mathbf{X}_{n}=\left(x_{n, 1}, x_{n, 2}, x_{n, 3}\right), 1 \leq n \leq v$, from the origin to the vertices of the polyhedron. In addition, consider a set of points $\mathcal{D}(\mathrm{A})$ in $v$-dimensional space $\mathfrak{R}^{v}, \mathbf{A}=\left(a_{1}, \ldots, a_{v}\right)$ such that the following sums vanish:

$$
\begin{gathered}
\sum_{i=1}^{v} a_{i} x_{i}, k=0 \text { for } 1 \leq k \leq 3 \\
\sum_{i=1}^{v} a_{i}=0
\end{gathered}
$$

Equation (1.11a) may also be viewed as three orthogonality relationships between the $v$-dimensional vector $\mathbf{A}=\left(a_{1}, \ldots, a_{v}\right)$ and the three $v$-dimensional vectors $\left(x_{1, k}, x_{2, k}, \ldots, x_{v, k}\right), 1 \leq k \leq 3$. Now consider the locations of the vertices of the polyhedron as the following $v \times 4$ matrix:

$$
\mathbf{D}_{0}=\left(\begin{array}{cccc}
x_{1,1} & x_{1,2} & x_{1,3} & 1 \\
x_{2,1} & x_{2,2} & x_{2,3} & 1 \\
\cdot & \cdot & \cdot & \\
\cdot & \cdot & \cdot & \\
x_{v, 1} & x_{v, 2} & x_{v, 3} & 1
\end{array}\right)
$$

Consider the columns of $\mathbf{D}_{0}$ as vectors in $\Re^{v}$. Since $\mathbf{D}_{0}$ has rank 4 , the four columns of $\mathbf{D}_{0}$ are linearly independent. Hence the subspace $\mathcal{M}(\mathrm{X})$ of $\mathfrak{R}^{v}$ represented by these four linearly independent columns has dimension 4 . Its orthogonal complement $\mathcal{M}(\mathbf{A})^{\perp}=\left\{\mathbf{A} \in \mathfrak{R}^{v} \mid \mathbf{A} \cdot \mathbf{X}=0\right.$ for all $\left.\mathbf{X} \in \mathcal{M}(\mathbf{X})\right\}$ coincides with $\mathcal{D}(\mathbf{A})$ defined above by equations (1.11a) and (1.11b). Therefore:

$$
\operatorname{dim} \mathcal{D}(\mathbf{A})=\operatorname{dim} \mathcal{M}(\mathbf{A})^{\perp}=v-\operatorname{dim} \mathcal{M}(\mathbf{X})=v-4
$$

Now define the following $v \times(v-4)$ matrix:

$$
\mathbf{D}_{1}=\left(\begin{array}{cccccc}
a_{1,1} & a_{1,2} & \cdot & \cdot & \cdot & a_{1, v-4} \\
a_{2,1} & a_{2,2} & \cdot & \cdot & \cdot & a_{2, v-4} \\
\cdot & \cdot & & & \cdot \\
\cdot & \cdot & & & \cdot \\
a_{v, 1} & a_{v, 2} & \cdot & \cdot & \cdot & a_{v, v-4}
\end{array}\right)
$$


The $v$ rows of $\mathbf{D}_{1}$ may be considered as vectors in ( $\left.v-4\right)$-dimensional space; conventionally the $j$ th row is denoted by $\bar{x}_{j}=\left(a_{i, 1}, a_{j, 2}, \ldots, a_{j, v-4}\right)$ for $j=$ $1, \ldots, v$.

The final result of this construction is the assignment of a point $\bar{x}_{j}$ in (v-4)dimensional space $\left(\mathfrak{R}^{v-4}\right)$ to each vertex $x_{j}$ of the polyhedron. The collection of $v$ points $\bar{x}_{1}, \ldots, \bar{x}_{v}$ in $\Re_{v-4}$ is called a Gale transform of the set of vertices $x_{1}, \ldots, x_{v}$ of the polyhedron in question. The following features of a Gale transform of a polyhedron should be noted:

(1) Gale transforms $\bar{x}_{j}$ and $\bar{x}_{k}$ of two or more vertices of a polyhedron may lead to the same point (i.e., the same $v-4$ coordinates) in ( $v-4)$-dimensional space $\left(\Re^{v-4}\right)$. In other words some points of a Gale transform may have a multiplicity greater than one so that the Gale transform of a polyhedron in such cases contains fewer distinct points than the polyhedron has vertices.

(2) The Gale transform depends upon the location of the origin in the coordinate system. Therefore, infinitely many Gale transforms are possible for a given polyhedron. Geometrically a Gale transform of a polyhedron corresponds to a projection of the $v$ vertices of a $(v-1)$-dimensional simplex [53]. Since infinitely many such projections are possible, the Gale transform for a given polyhedron is not unique.

In practice, it is easier to work with Gale diagrams corresponding to Gale transforms of interest. Consider a Gale transform of a (three-dimensional) polyhedron having $v$ vertices $\bar{x}_{1}, \ldots, \bar{x}_{v}$ as defined above. The corresponding Gale diagram $\hat{x}_{1}, \ldots, \hat{x}_{v}$ is defined by the following relationships:

$$
\begin{array}{ll}
\hat{x}_{i}=0 \quad \text { if } & \bar{x}_{i}=0 \\
\hat{x}_{i}=\frac{x_{i}}{\left\|\bar{x}_{i}\right\|} & \text { if } \quad \bar{x}_{i} \neq 0
\end{array}
$$

In Equation (1.15b) $\left\|\bar{x}_{i}\right\|$ is the length (i.e., $\sqrt{a_{i, 1}^{2}+a_{i, 2}^{2}+\cdots+a_{i, v-4}^{2}}$ ) of the vector $\bar{x}_{i}$. If $v-4=1$ (i.e., $v=5$ ), Gale diagrams can only contain the points of the straight line 0,1 , and -1 of varying multiplicities $m_{0}, m_{1}$, and $m_{-1}$, respectively, where $m_{0} \geq 0, m_{1} \geq 2$, and $m_{-1} \geq 2$. If $v-4=2$ (i.e., $v=6$ ) Gale diagrams can only contain the center and circumference of the unit circle. These two types of Gale diagrams (Figure 1.9) are of interest for the study of polyhedral isomerizations since they represent significant structural simplifications of the corresponding polyhedra.

The following properties of Gale diagrams corresponding to threedimensional polyhedra are of interest since they impose important restrictions on configurations of points which can be Gale diagrams:

(1) Any ( $v-5)$-dimensional plane passing through the central point of the Gale diagram bisects the space of the Gale diagram into two halfspaces. Each 
(a)

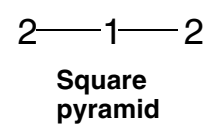

$2-3$

Trigonal

(b)

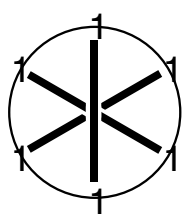

Trigonal prism

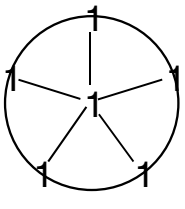

Pentagonal pyramid

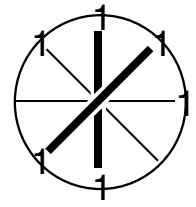

$C_{2}$ 6-vertex polyhedron with 2 quadrilateral faces
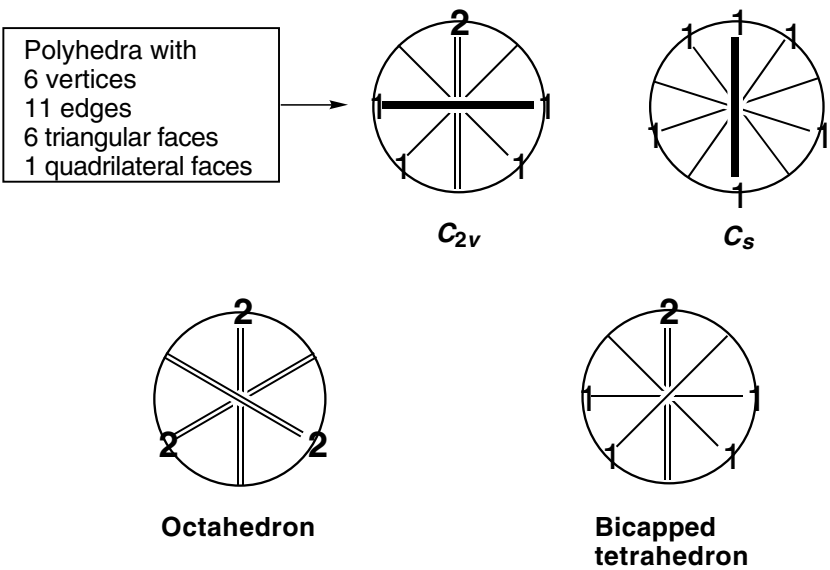

Figure 1.9 (a) Gale diagrams for the two five-vertex polyhedra. (b) Standard Gale diagrams for the seven six-vertex polyhedra.

such halfspace must contain at least two vertices (or one vertex of multiplicity 2) of the Gale diagram not including any vertices actually in the bisecting plane or hyperplane. Such a halfspace is called an open halfspace. Violation of this condition corresponds to a polyhedron with the impossible property of at least one pair of vertices not connected by an edge which is closer in three-dimensional space than another pair of vertices which is connected by an edge.

(2) The set of vertices of a polyhedron not forming a given face or edge of the polyhedron is called a coface of the polyhedron. The regular octahedron is unusual since all of its faces are also cofaces corresponding to other faces. The interior of a figure formed by connecting the vertices of a Gale diagram corresponding to a coface must contain the central point. 
(3) The central point is a vertex of a Gale diagram if and only if the corresponding polyhedron is a pyramid. The central vertex of such a Gale diagram corresponds to the apex of a pyramid which is the coface corresponding to the base of the pyramid.

Nonplanar isomerizations of five- and six-vertex polyhedra correspond to allowed vertex motions in the corresponding Gale diagrams in Figure 1.9. In this context an allowed vertex motion of a Gale diagram is the motion of one or more vertices which converts the Gale diagram of a polyhedron into that of another polyhedron with the same number of vertices without ever passing through an impossible Gale diagram. Such impossible Gale diagrams include those with an open halfspace containing only one vertex of unit multiplicity. Since two polyhedra are combinatorially equivalent if and only if their Gale diagrams are isomorphic, such allowed vertex motions of Gale diagrams are faithful representations of all possible non-planar polyhedral isomerizations.

The application of Gale diagrams to the study of isomerizations of five-vertex polyhedra is nearly trivial but provides a useful illustration of this method. The only possible five-vertex polyhedra are the square pyramid and trigonal bipyramid. Their Gale diagrams (Figure 1.9a) are the only two possible onedimensional five-vertex Gale diagrams which have the required two vertices in each open halfspace (i.e., $m_{1} \geq 2$ and $m_{-1} \geq 2$ ). The only allowed vertex motion in a Gale diagram of a trigonal bipyramid involves motion of one point from the vertex of multiplicity 3 through the center point to the vertex originally of multiplicity 2 as indicated in Figure 1.6c. This process interchanges the vertices of multiplicities 2 and 3 and leads to an equivalent Gale diagram corresponding to an isomeric trigonal bipyramid. The motion through the center point of the Gale diagram corresponds to the generation of a square pyramid intermediate in the nonplanar degenerate isomerization of a trigonal bipyramid. This, of course, is the Berry pseudorotation process $[39,44]$ that is the prototypical dsd process. The choice of three points to move away from the vertex of multiplicity 3 in the Gale diagram of a trigonal bipyramid corresponds to the presence of three degenerate edges in a trigonal bipyramid. This analysis of the Gale diagrams of the two possible five-vertex polyhedra shows clearly that the only possible nonplanar isomerizations of five-vertex polyhedra can be represented as successive dsd processes corresponding to successive Berry pseudorotations (Figure 1.6).

The Gale diagrams of six-vertex polyhedra (Figure 1.9b) can be visualized most clearly if all of the diameters containing vertices are drawn. Some Gale diagrams of six vertex polyhedra have diameters with vertices of unit multiplicity at each end. Such diameters may be called balanced diameters and are indicated by bold lines in Figure 1.9b. The two vertices of a balanced diameter in the Gale diagram of a six-vertex polyhedron form an edge which is a coface corresponding to a quadrilateral face. Gale diagrams drawn to maximize the 
multiplicities of the vertices and the numbers of balanced diameters consistent with the polyhedral topology are called standard Gale diagrams. The Gale diagrams depicted in Figure 1.9b are the standard Gale diagrams for the six-vertex polyhedra in question. The number of balanced diameters in a standard Gale diagram of a six-vertex polyhedron is equal to the number of quadrilateral faces of the polyhedron. The pentagonal pyramid is the only six-vertex polyhedron for which the center of the circle is a vertex of the corresponding standard Gale diagram.

The standard Gale diagrams of the trigonal prism and octahedron illustrate another interesting feature of Gale diagrams, namely the ability to draw Gale diagrams so that all symmetry elements of the corresponding polyhedron are preserved. The $C_{3}$ symmetry elements of both the trigonal prism and octahedron are readily apparent in their standard Gale diagrams passing through the center perpendicular to the plane of the circle (Figure 1.9b). In the case of the trigonal prism, the three $C_{2}$ axes of its $D_{3 h}$ point group correspond to the three balanced diameters of the corresponding standard Gale diagram. In the case of the octahedron, which has the $O_{h}$ point group, the reflection planes $\sigma_{h}$ correspond to permuting the two vertices of an octahedron forming a vertex of multiplicity two in the corresponding standard Gale diagram while keeping the other vertices fixed. The $C_{2}$ and $C_{4}$ rotation axes of the octahedron pass through the center and a vertex of multiplicity two in the corresponding standard Gale diagram and permute the other four vertices forming the two other standard Gale diagram vertices of multiplicity two in various ways.

Alternatively the regular octahedron can be considered to be a regular $\mathrm{K}_{2,2,2}$ tripartite graph [54]. Thus the six vertices of the octahedron are partitioned into three antipodal pairs (i.e., two vertices with a mutual trans relationship). Each vertex then has an edge to every vertex other than its antipode in accord with the definition of a multipartite graph [51]. Each of the three double points in the Gale diagram of a regular octahedron (Figure 1.9b) corresponds to one of the three antipodal pairs of vertices.

Polyhedral isomerizations in six-vertex polyhedra may be described by allowed motions of the vertices of their Gale diagrams along the circumference of the unit circle or through the circle center in the case of polyhedral isomerizations involving a pentagonal pyramid intermediate. However, vertex motions are not allowed if at any time they generate one or more forbidden diameters containing three or more vertices. Using these techniques all nonplanar degenerate isomerizations of six-vertex polyhedra can be decomposed into sequences of eight fundamental processes, namely two processes through pentagonal pyramid intermediates, five processes which are variations of single diamond-square-diamond processes, and the triple dsd degenerate isomerization of an octahedron through a trigonal prism intermediate on which the Bailar [55] and Ray and Dutt [56] twists of M(bidentate) ${ }_{3}$ complexes are based. 


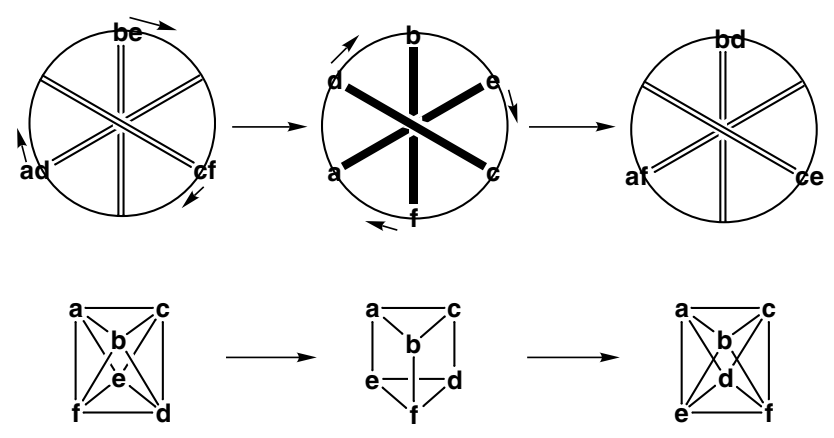

\section{Octahedron $\longrightarrow$ Trigonal prism $\longrightarrow$ Octahedron}

Figure 1.10 Gale diagrams for the triple dsd degenerate isomerization of an octahedron through a trigonal prismatic intermediate corresponding to the Bailar or Ray/Dutt twists.

The Gale diagrams depicting the latter processes are depicted in Figure 1.10. Note that in the first (diamond-square) stage of this triple dsd process leading from the octahedron to the trigonal prism, one vertex from each of the three vertex pairs (i.e., $a d$, $b e$, and $c f$ ) in the Gale diagram of the octahedron must move in the same direction in a concerted manner preserving the $C_{3}$ axis in order to avoid violating the 'half-space rule.' The standard Gale diagram of the trigonal prism is reached when three balanced diameters are formed. Similarly, in the second (square-diamond) stage leading from the trigonal prism to an isomeric octahedron these three vertices continue to move in a concerted manner so as to preserve the $C_{3}$ axis.

\subsection{GENERATION OF METALLABORANE STRUCTURES BY DIAMOND-SQUARE-DIAMOND TRANSFORMATIONS IN DELTAHEDRA}

The basic building blocks of the most stable boranes, namely $\mathrm{B}_{n} \mathrm{H}_{n}{ }^{2-}(6 \leq n \leq$ 12) and their isoelectronic carborane analogues $\mathrm{CB}_{n-1} \mathrm{H}_{n}{ }^{-}$and $\mathrm{C}_{2} \mathrm{~B}_{n-2} \mathrm{H}_{n}$, are the most spherical deltahedra (Figure 1.4). In these deltahedra all of the vertices have degrees 4 or 5 except for the topologically required single degree 6 vertex [57] in the $\mathrm{B}_{11} \mathrm{H}_{11}{ }^{2-}$ deltahedron. The boron vertices in these deltahedra can be replaced by isolobal transition metal vertices bearing sufficient external ligands such as carbonyl groups or perhapto planar polygons (e.g., $\eta^{5}-\mathrm{C}_{5} \mathrm{H}_{5}$ or $\eta^{6}-\mathrm{C}_{6} \mathrm{H}_{6}$ ) to give the transition metal vertex a favorable electronic configuration. Initially it was assumed that the deltahedra in metallaboranes would be the same as the deltahedra in isoelectronic metal-free boranes. However, as metallaborane chemistry was developed further, particularly by Kennedy and co-workers [58-61], a variety of deltahedral metallaborane structures were 
discovered based on deltahedra topologically distinct from the most spherical deltahedra found in simple metal-free boranes and carboranes (Figure 1.4). Of particular interest was the discovery of 9- and 10-vertex metallaboranes based on deltahedra with the transition metal at a degree 6 vertex whereas the metalfree borane has a most spherical deltahedral structure (Figure 1.4) with only degree 4 and 5 vertices.

The experimentally observed metallaborane deltahedra are related to the most spherical deltahedra by a series of one or more dsd processes [62,63]. For example, among the 10-vertex deltahedra a single dsd process converts the most spherical bicapped square antiprism (Figure 1.4) of four-fold $D_{4 d}$ symmetry found in $\mathrm{B}_{10} \mathrm{H}_{10}{ }^{2-}$ to a deltahedron of three-fold $C_{3 v}$ symmetry with a single degree 6 vertex (Figure 1.11a). The 'square' intermediate in this single

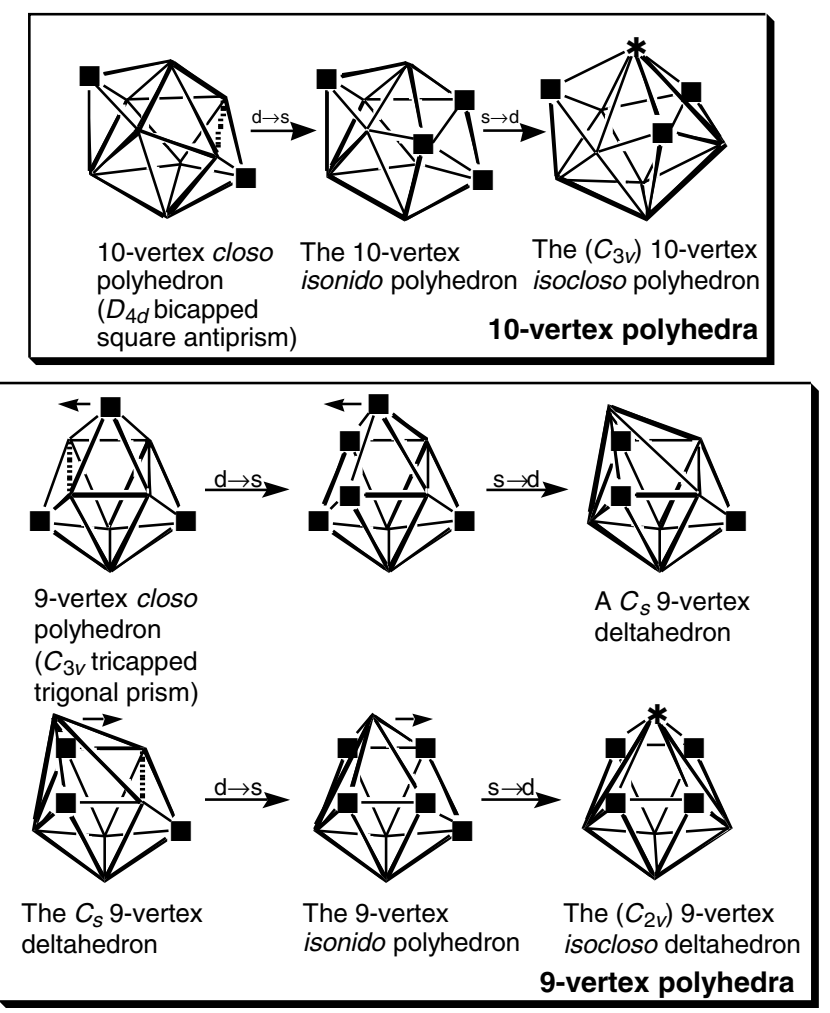

Figure 1.11 (a) Conversion of a $D_{4 d}$ bicapped square antiprism to the $C_{3 v}$ isocloso 10vertex deltahedron through a dsd process. (b) Conversion of a $C_{3 v}$ tricapped trigonal prism to a $C_{2 v}$ isocloso 9 -vertex deltahedron through a sequence of two dsd processes. For clarity the vertices of degrees $3,4,6$, and 7 are labelled with the symbols $\boldsymbol{\Lambda}, \mathbf{\square}, *$, and $\boldsymbol{\theta}$, respectively, in Figures 1.11 and 1.12. The vertices of degree 5 are not labelled in these figures. 


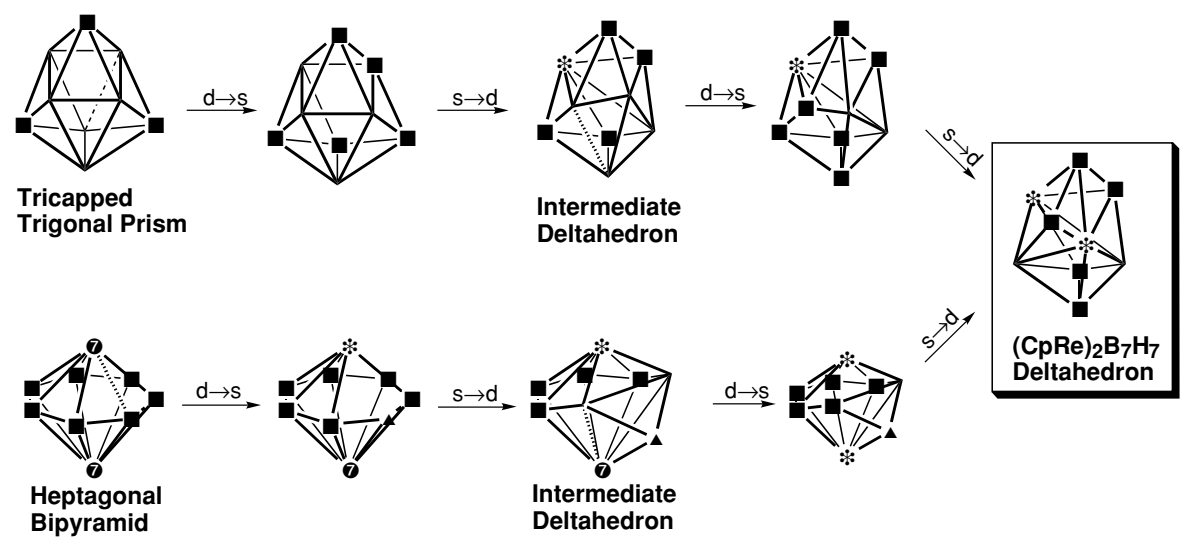

Figure 1.12 Two ways of generating the unusual 9-vertex deltahedron found in $\left(\eta^{5}-\mathrm{C}_{5} \mathrm{H}_{5} \mathrm{Re}\right)_{2} \mathrm{~B}_{7} \mathrm{H}_{7}$ by sequences of two dsd processes: (a) from a $D_{3 h}$ tricapped trigonal prism; (b) from a $D_{7 h}$ heptagonal bipyramid.

dsd process is a so-called 10-vertex isonido polyhedron with a single quadrilateral face. In metallaboranes the transition metal occupies the degree 6 vertex. Similarly among the 9-vertex deltahedra two successive dsd processes convert the most spherical tricapped trigonal prism (Figure 1.4) to a $C_{2 v}$ deltahedron with a degree 6 vertex for a transition metal (Figure 1.11b). This 9-vertex deltahedron is observed experimentally in $\left(\mathrm{Me}_{3} \mathrm{P}\right)_{2} \mathrm{HIrB}_{8} \mathrm{H}_{7} \mathrm{Cl}$ [64] with the iridium atom at the degree 6 vertex.

The conversion of a most spherical borane $n$-vertex deltahedron to a deltahedron with a degree 6 vertex for a transition metal atom leads to a change in its skeletal electron count from $2 n+2$ to simply $2 n$ [58-61]. This has been attributed to a change in the skeletal bonding from a globally delocalized model with an $n$-center core bond to a more localized bonding model with two-center bonds along some of the deltahedral edges and three-center bonds in some of the deltahedral faces [62].

Two different sequences of dsd processes can be used to generate the rather unusual 9-vertex deltahedron found in $\left(\eta^{5}-\mathrm{C}_{5} \mathrm{H}_{5} \mathrm{Re}\right)_{2} \mathrm{~B}_{7} \mathrm{H}_{7}$ with the rhenium atoms at the two degree 6 vertices (Figure 1.12) [65]. In the first such sequence, this deltahedron is obtained by a sequence of two dsd processes from the most spherical 9-vertex deltahedron, namely the tricapped trigonal prism (Figure 1.12a). This double dsd sequence generates two degree 6 vertices from a deltahedron having only degree 4 and 5 vertices. Alternatively, $\left(\eta^{5}-\mathrm{C}_{5} \mathrm{H}_{5} \mathrm{Re}\right)_{2} \mathrm{~B}_{7} \mathrm{H}_{7}$ can be obtained by a sequence of two dsd process from the heptagonal bipyramid (Figure 1.12b). This double dsd sequence eliminates the two degree 7 vertices from the heptagonal bipyramid leaving two degree 6 vertices for the rhenium atoms. 


\section{POLYHEDRAL ISOMERIZATIONS: MACROSCOPIC MODELS}

\subsection{TOPOLOGICAL REPRESENTATIONS OF POLYHEDRAL ISOMERIZATIONS}

Macroscopic models depict the relationship between different permutational isomers. Such models make use of topological representations (top-reps), which are reaction graphs $[66,67]$ describing the relationships between the different permutational isomers of a given polyhedron. Thus, consider an $\mathrm{ML}_{n}$ compound having $n$ ligands $\mathrm{L}$ around a central metal $\mathrm{M}$ or a cluster compound having $n$ vertices. There are a total of $n$ ! permutations of the ligand sites or the cluster vertices. These permutations form a group of order $n$ ! called the symmetric group $[68,69]$ and conventionally designated as $S_{n}$. The symmetric group $S_{n}$ is the automorphism group corresponding to the symmetry of the complete graph $K_{n}$ [51].

Now consider the symmetry point group $G$ (or, more precisely, the framework group [70]) of the above $\mathrm{ML}_{n}$ coordination compound or $n$-vertex cluster compound. This group has $|G|$ operations of which $|R|$ are proper rotations so that $|G| /|R|=2$ if the compound is achiral and $|G| /|R|=1$ if the compound is chiral (i.e., has no improper rotations). The $n$ ! distinct permutations of the $n$ sites in the coordination compound or cluster are divided into $n ! /|R|$ right cosets [71] which represent the permutational isomers since the permutations corresponding to the $|R|$ proper rotations of a given isomer do not change the isomer but merely rotate it in space. This leads naturally to the concept of isomer count, I, namely

$$
I=n ! /|R|
$$

if all vertices are distinguishable. Similarly the quotient

$$
E=n ! /|G|=I / 2
$$

for a given chiral polyhedron corresponds to the number of enantiomeric pairs.

Top-reps are reaction graphs [66,67] describing the relationships between polyhedral isomers. They there have $I$ (Equation 1.16) or $E$ vertices (Equation 1.17) depending upon whether individual isomers or enantiomeric pairs, respectively, are considered. The degree of a vertex corresponds to the number of new permutational isomers generated from the isomer represented by the vertex in a single step. This is called the connectivity, $\delta$, of the vertex. Such reaction graphs for degenerate polyhedral isomerizations have been shown to be vertex- and edge-transitive [72].

The top-reps for the isomerizations of four- and five-vertex molecular polyhedra are quite straightforward. Thus the top-rep for four-vertex isomerizations is a graph with two degree 3 vertices and three degree 2 vertices corresponding to the unsymmetrical bipartite graph $K_{2,3}$ or to a trigonal bipyramid with the 


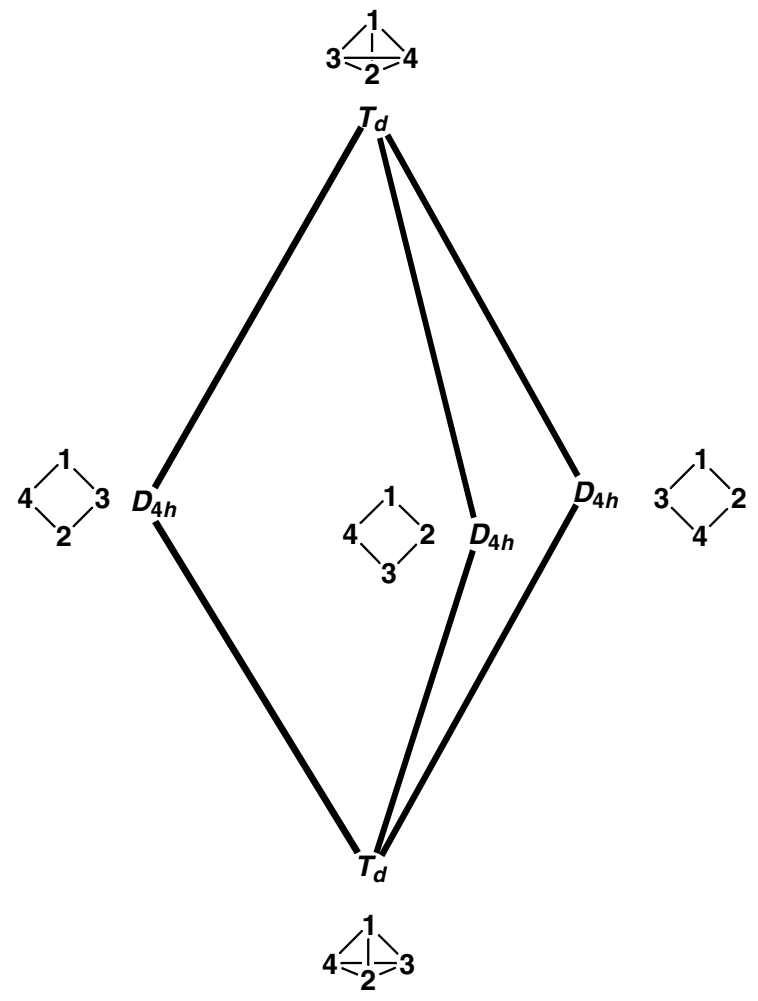

Figure 1.13 The $K_{2,3}$ bipartite graph top-rep describing isomerization of four-vertex molecular polyhedra in which the sites marked $T_{d}$ and $D_{4 h}$ correspond to tetrahedral and square planar isomers, respectively. The isomers corresponding to the vertices of the $K_{2,3}$ bipartite graph are depicted next to the vertex labels.

equatorial-equatorial edges missing (Figure 1.13). The two degree 3 vertices (the axial vertices of the underlying trigonal bipyramid) correspond to the two tetrahedral isomers $\left(I_{\mathrm{tet}}=4 ! /|T|=24 / 12=2\right)$. Similarly, the three degree 2 vertices (the equatorial vertices of the underlying trigonal bipyramid) correspond to the three square planar isomers $\left(I_{\mathrm{sq}}=4 ! /\left|D_{4}\right|=24 / 8=3\right)$. The connectivities of the tetrahedral $\left(\delta_{\mathrm{tet}}\right)$ and square planar $\left(\delta_{\mathrm{sq}}\right)$ isomers are 3 and 2 , respectively, in accord with the corresponding degrees of the $K_{2,3}$ graph. Thus $I_{\mathrm{tet}} \delta_{\mathrm{tet}}=I_{\mathrm{sq}} \delta_{\mathrm{sq}}=6$. This is an example of the closure condition $I_{a} \delta_{a}=I_{b} \delta_{b}$ required for a top-rep representing more than one type of polyhedron.

In order to consider the top-reps of more complicated polyhedra, it is first instructive to consider the top-rep of four-vertex isomerizations depicted in Figure 1.13 in a different way, namely as a graph with only two vertices connected by three edges like the hydrogen-deleted molecular graph of acetylene, $\mathrm{HC} \equiv \mathrm{CH}$. Thus each vertex is of degree 3 and the two vertices correspond 


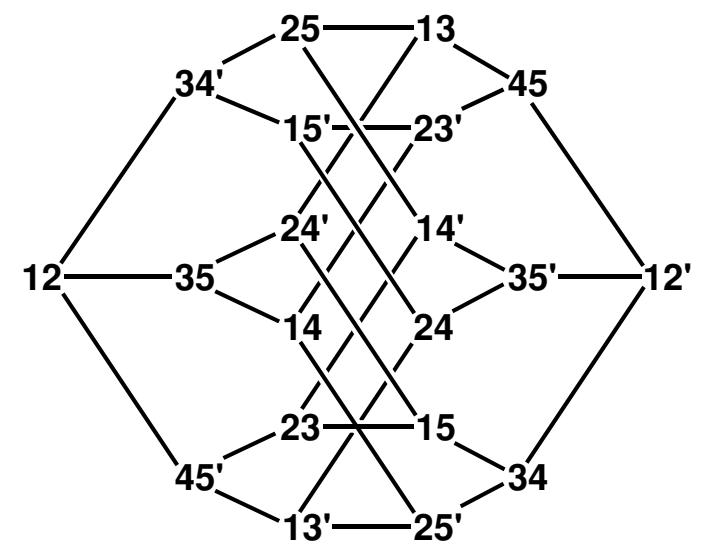

\section{0-Vertex Desargues-Levy Graph}

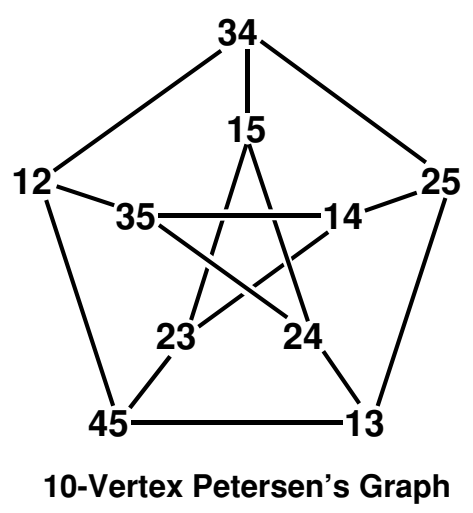

Figure 1.14 (a) The 20-vertex Desargues-Levy graph as a top-rep of the dsd isomerizations of the 20 trigonal bipyramid isomers through Berry pseudorotation processes (Figure 1.6). (b) The 10-vertex Petersen graph as a top-rep of the dsd isomerizations of the 10 trigonal bipyramid enantiomer pairs.

to the two tetrahedral isomers. The midpoints of the three edges correspond to the square planar isomers. Using this approach the top-rep of fivevertex isomerizations is a 20 -vertex Desargues-Levy graph with the 20 vertices representing the trigonal bipyramid isomers, i.e. $I_{\mathrm{tbp}}=5 ! /\left|D_{3}\right|=120 / 6=20$ (Figure 1.14a). The midpoints of the 30 edges represent the square pyramid isomers, i.e., $I_{\mathrm{sp}}=5 ! /\left|C_{4}\right|=120 / 4=30$. The connectivities of the trigonal bipyramidal isomers $\left(\delta_{\mathrm{tbp}}\right)$ are 3 in accord with their locations at degree 3 vertices in the corresponding top-rep. Similarly, the connectivities of the square pyramidal isomers $\left(\delta_{\mathrm{sp}}\right)$ are 2 in accord with their locations at edge midpoints of the top-rep. As in the case of four-vertex isomerizations, the closure condition is 
met for five-vertex isomerization since $I_{\mathrm{sp}} \delta_{\mathrm{sp}}=I_{\mathrm{tbp}} \delta_{\mathrm{tbp}}=6$. Travelling from one vertex to another in the Desargues-Levy graph (Figure 1.14a) corresponds to a Berry pseudorotation process [39,44] (Figure 1.6) through the square pyramidal intermediate represented by the edge midpoint.

Less complicated but still useful top-reps can be obtained by using each vertex of the graph to represent a set of isomers provided that each vertex represents sets of the same size and interrelationship and each isomer is included in exactly one set. A simple example is the use of the Petersen graph (Figure 1.14b) as a toprep of the ten trigonal bipyramid enantiomer pairs $\left(E=5 ! /\left|D_{3 h}\right|=120 / 12=\right.$ $10)$ by dsd processes. The use of the Petersen graph for this purpose relates to its being the odd graph $O_{3}$. In this connection, an odd graph $O_{k}$ has vertices corresponding to subsets of cardinality $k-1$ of a set $S$ of cardinality $2 k-1$ (an odd number). Two vertices of $O_{k}$ are adjacent if and only if the corresponding subsets are disjoint.

In six-vertex structures the process of interest is the degenerate triple dsd isomerization of the octahedron through a trigonal prismatic intermediate which is the underlying topology of both the Bailar [55] and Ray and Dutt [56] twists for $\mathrm{M}$ (bidentate) ${ }_{3}$ chelates. The isomer counts are $I_{\text {oct }}=6 ! /|O|=720 / 24=30$ for the octahedron and $I_{\mathrm{tp}}=6 !\left|D_{3}\right|=720 / 6=120$ for the trigonal prism. A pentagonal $\left(I_{h}\right)$ dodecahedron in double group form [1] can serve as the top-rep for this process (Figure 1.15). The midpoints of the 30 edges of the dodecahedron (designated by triangles in Figure 1.15) are the 30 octahedron isomers. Line segments across a pentagonal face connecting these edge midpoints correspond to triple dsd isomerization processes. The midpoints of these lines (designated by diamonds) correspond to the 120 trigonal prismatic isomers with ten such isomers being located in each of the 12 faces of the pentagonal dodecahedron. The ten lines on a face representing isomerization processes form a $K_{5}$ graph. This system is closed since the connectivities of the octahedron $\left(\delta_{o c t}\right)$ and trigonal prism $\left(d_{\mathrm{tp}}\right)$ are 8 and 2 , respectively, leading to the closure relationship $I_{\text {oct }} \delta_{\text {oct }}=I_{\mathrm{tp}} \delta_{\mathrm{tp}}=240$.

Development of top-reps for isomerizations of polyhedra having more than six vertices is complicated by large isomer counts. Thus for the most symmetrical and chemically significant seven-vertex deltahedra the isomer counts are $7 ! /\left|D_{5}\right|=5040 / 10=504$ for the pentagonal bipyramid and $7 ! /\left|C_{3}\right|=$ $5040 / 3=1680$. In this connection a 'baby monster' graph with 1680 vertices has actually been described in the chemical literature as a model for degenerate rearrangements in the seven-vertex $\mathrm{P}_{7}^{3-}$ system [73].

Among polyhedra with eight vertices the isomer counts of the cube, hexagonal bipyramid, square antiprism, and bisdisphenoid are $40320 / 24=1680$, $40320 / 12=3360,40320 / 8=5040$, and $40320 / 4=10080$, respectively. Graphs corresponding to top-reps in systems with such large numbers of polyhedral isomers are clearly unwieldy and unmanageable. However, the problem of representing permutational isomerizations in seven- and eight-vertex polyhedra can be simplified if subgroups of the symmetric groups $S_{n}(n=7,8)$ can be 


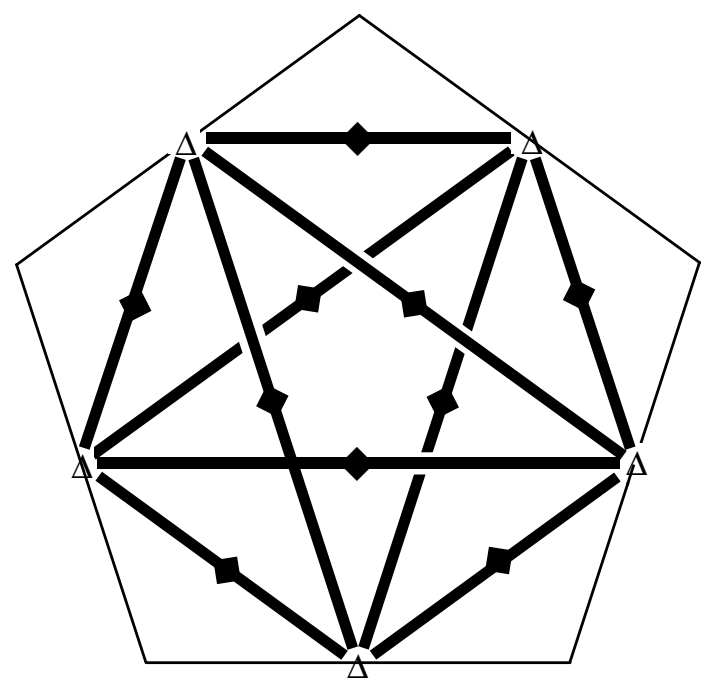

Figure 1.15 One of the 12 pentagonal faces of the $I_{h}$ double group pentagonal dodecahedron used as a topological representation for the degenerate triple dsd degenerate isomerization of the octahedron through a trigonal prismatic intermediate (see Figure 1.10). The five triangles $(\Delta)$ at the midpoints of the sides of the face represent five of the 30 octahedron permutational isomers. The ten diamonds $(\bullet)$ at the midpoints of the edges of the $K_{5}$ graph drawn on the face represent 10 of the 120 trigonal prism permutational isomers.

found which contain all of the symmetries of all of the polyhedra of interest. This is not possible for the seven-vertex system since there is no subgroup of $S_{7}$ that contains both the five-fold symmetry of the pentagonal bipyramid and the three-fold symmetry of the capped octahedron. The situation with the eightvertex system is more favorable since the wreath product group [74-76] $S_{4}\left[S_{2}\right]$ of order 384 contains all of the symmetries of the cube, hexagonal bipyramid, square antiprism, and bisdisphenoid [77], which are all of the eight-vertex polyhedra of actual or potential chemical interest. The major effect of reducing the symmetry by a factor of $105(3 \times 5 \times 7)$ in going from $S_{8}$ to $S_{4}\left[S_{2}\right]$ is the deletion of five-fold and seven-fold symmetry elements. Such symmetry elements are not of interest in this context since none of the 257 eight-vertex polyhedra has fivefold symmetry elements $[32,33]$ and the only eight-vertex polyhedron having a seven-fold symmetry element is the heptagonal pyramid, which is not of interest in this particular chemical context. Restricted isomer counts $I^{*}=384 /|R|$ based on subgroups of the wreath product group $S_{4}\left[S_{2}\right]$ rather than the symmetric group $S_{8}$ are the more manageable numbers $16,32,48$, and 96 for the cube, hexagonal bipyramid, square antiprism, and bisdisphenoid, respectively [78].

The concept of restricting vertex permutations in eight-vertex systems to the wreath product group $S_{4}\left[S_{2}\right]$ rather than the fully symmetric $S_{8}$ group can 
be restated in graph-theoretical terms using the hyperoctahedral graph $H_{4}$ [79]. Therefore such a restriction of permutations from $S_{8}$ to $S_{4}\left[S_{2}\right]$ can be called a hyperoctahedral restriction. The hyperoctahedral graphs underlying this restriction are designated as $H_{n}$ and have $2 n$ vertices and $2 n(n-1)$ edges with every vertex connected to all except one of the remaining vertices so that each vertex of $H_{n}$ has degree $2(n-1)$. The name 'hyperoctahedral' comes from the fact that an $H_{n}$ graph is the 1-skeleton of the analogue of the octahedron (called the 'cross-polytope') in $n$-dimensional space [80-82]. The hyperoctahedral graphs $\mathrm{H}_{2}$ and $\mathrm{H}_{3}$ thus correspond to the square and octahedron, respectively. The $S_{4}\left[S_{2}\right]$ wreath product group is the automorphism ('symmetry') group of the hyperoctahedral graph $H_{4}$ just as the $S_{8}$ symmetric group is the automorphism group of the complete graph $K_{8}$.

These considerations lead to a top-rep for isomerizations of eight-vertex polyhedra based on a $K_{4,4}$ bipartite graph of hexagons (Figure 1.16) with special points corresponding to the individual eight-coordinate polyhedra as follows:

(1) The eight hexagons at the vertices of the $K_{4,4}$ bipartite graph (Figure 1.16a) correspond to the eight cube isomers.

(2) The 16 edge midpoints of the $K_{4,4}$ bipartite graph (Figure 1.16a) correspond to the 16 hexagonal bipyramid isomers.

(3) The 48 vertices of the eight hexagons linked by the $K_{4,4}$ bipartite graph (Figure 1.16b) correspond to the 24 square antiprism isomers.

(4) The 48 edge midpoints of the eight hexagons linked by the $K_{4,4}$ bipartite graph (Figure 1.16b) correspond to the 48 bisdisphenoid isomers.

Moving along the circumference of a given hexagon corresponds to a sequence of double dsd processes interconverting the bisdisphenoids located at the midpoints of the two joined hexagonal edges meeting at a vertex. The vertex common to these two joined hexagonal edges corresponds to the square antiprism intermediate in this double dsd process. Since both the bisdisphenoid and square antiprism can be formed using only s, $p$, and d orbitals, the circumference of the hexagon is accessible in $\mathrm{ML}_{8}$ systems in which the central atom $\mathrm{M}$ has the usual $\mathrm{sp}^{3} \mathrm{~d}^{5}$ nine-orbital manifold. Thus in the usual situation not involving $\mathrm{f}$ orbitals, isomerizations are restricted to the circumference of a given hexagon in Figure 1.16b and cannot occur by moving from one hexagon to another along the edges of the $K_{4,4}$ bipartite graph (Figure 1.16a).

The group-theoretical aspects of top-reps can be generalized by considering a set of groups designated as $G$ and $H_{1}, \ldots, H_{n}$ where the following apply: (1) $H_{1}, \ldots, H_{n}$ are symmetry point groups which are all subgroups of $G$; (2) $\left|H_{1}\right| \geq\left|H_{2}\right| \geq \ldots \geq\left|H_{n}\right|$; (3) $R_{m}$ is the rotation subgroup of $H_{m}$. If $n=2$, then we can seek a graph in which the number of vertices is $|G| /\left|H_{1}\right|$ and the number of edges is $|G| /\left|H_{2}\right|$. Such is the case with the $K_{2,3}$ top-rep of four-vertex isomerizations where $G=S_{4}\left(\approx T_{d}\right), H_{1}=T_{d}$, and $H_{2}=D_{4 h}$ (Figure 1.13) and 

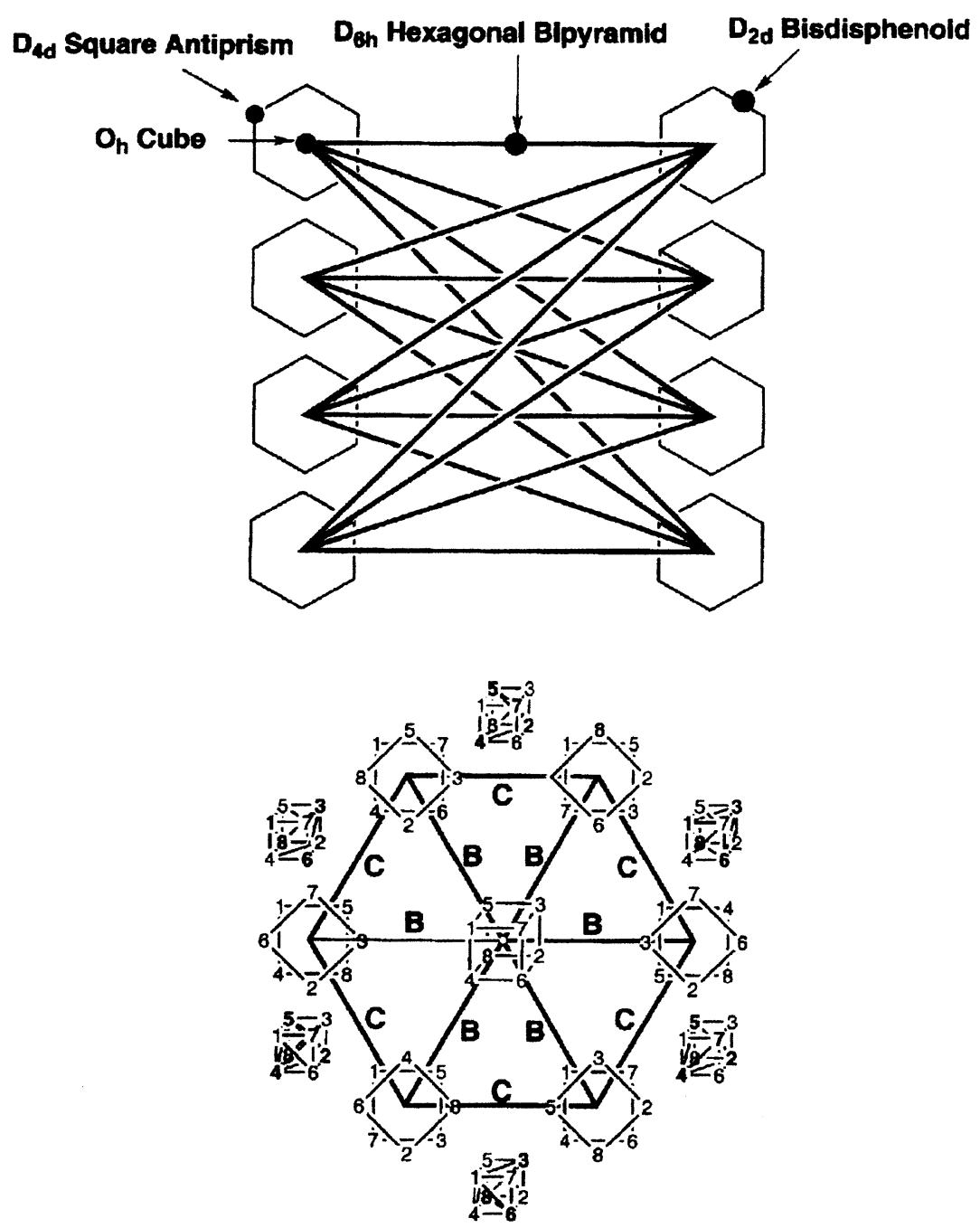

Figure 1.16 (a) The $K_{4,4}$ graph of hexagons used as a top-rep for hyperoctahedrally restricted isomerizations involving four types of eight-vertex polyhedra, namely the cube, hexagonal bipyramid, square antiprism, and $D_{2 d}$ bisdisphenoid. (b) Details of a hexagon in the top-rep in Figure 1.16a.

with the Desargues-Levy graph top-rep of five-vertex isomerizations where $G=S_{5}, H_{1}=D_{3 h}$, and $H_{2}=C_{4 v}$ (Figure 1.12a). In the case of the hyperoctahedrally restricted eight-vertex isomerizations with the $K_{4,4}$ graph of hexagons as the corresponding top-rep (Figure 1.16), $G$ is not $S_{8}$ but instead $S_{4}\left[S_{2}\right], H_{1}=O_{h}$ (for the cube), $H_{2}=D_{6 h}$ (for the hexagonal bipyramid), $H_{3}=D_{4 d}$ (the square antiprism), and $H_{4}=D_{2 d}$ (the bisdisphenoid) [78]. 


\subsection{TOPOLOGICAL REPRESENTATIONS OF JAHN-TELLER DISTORTIONS}

\subsubsection{Background}

The Jahn-Teller (JT) theorem asserts the existence of spontaneous symmetry breaking distortions in degenerate electronic states of nonlinear molecules [2124]. However, the amount and type of symmetry breaking is restricted by the epikernel principle $[83,84]$. As a result of the epikernel principle JT distortions leading to stable minima on the potential energy surface near an electronic degeneracy preserve larger symmetry groups than would be expected from an unrestricted action of the JT distorting forces. The relationships between possible JT distorted structures can be depicted by top-reps similar to those discussed in the previous section [20].

The underlying group theory for the study of JT distortions uses a direct product $[85,86] \Gamma \times \Gamma$ of a suitable irreducible representation (irrep) $\Gamma$ of the symmetry group $G$ of the undistorted nuclear configuration. The characters of $\Gamma \times \Gamma$ are the squares of the characters of the corresponding operations of $G$ in the irrep $\Gamma$ according to the following equation where $g$ is any operation in $G$ :

$$
\chi(g: \Gamma \times \Gamma)=(\chi(g: \Gamma))^{2}
$$

If the dimension of $\Gamma$ is $d$, then the dimension of $\Gamma \times \Gamma$ is $d^{2}$. Furthermore, the direct product representation $\Gamma \times \Gamma$ can be reduced into a sum of two lowerdimensional representations, namely the symmetric part $[\Gamma \times \Gamma]_{\mathrm{S}}$ of dimension $1 / 2 d(d+1)$ and the antisymmetric part $\{\Gamma \times \Gamma\}_{\mathrm{A}}$ of dimension $1 / 2 d(d-1)$. The characters of the symmetric and antisymmetric parts of the direct product $\Gamma \times \Gamma$ can be determined by the following formulas:

$$
\begin{aligned}
\chi\left(g:[\Gamma \times \Gamma]_{\mathrm{S}}\right) & =1 / 2\left[(\chi(g: \Gamma))^{2}+\chi\left(g^{2}: \Gamma\right)\right] \\
\chi\left(g:\{\Gamma \times \Gamma\}_{\mathrm{A}}\right) & =1 / 2\left[(\chi(g: \Gamma))^{2}-\chi\left(g^{2}: \Gamma\right)\right]
\end{aligned}
$$

According to the rule of the JT instability [87], the JT distortion coordinates of a degenerate electronic state corresponding to an irreducible representation (irrep) $\Gamma$ must span representations $\Lambda$ belonging to the non-totally symmetric part of the symmetrized direct product of $\Gamma$, i.e.

$$
\Lambda \in[\Gamma \times \Gamma]_{\mathrm{S}}-A_{1}
$$

Note that for a $d$-dimensional irrep $\Gamma$ the total dimension of the corresponding representation $\Lambda$ is $[d(d+1) / 2]-1$.

The reduction of the representation $\Lambda$ to a sum of irreps depends on the symmetry point group $G$ of the undistorted nuclear configuration. A system of JT distortions corresponding to a given $\Gamma$ in a point group $G$ is conventionally described [24] as a JT problem of the type $\Gamma \otimes \Lambda$ or $\Gamma \otimes \sum \lambda_{k}$ where $\Gamma$ is the representation of $G$ containing the degenerate electronic state and the $\lambda_{k} \mathrm{~s}$ are the irreps of $G$ contained in the representation $\Lambda$ defined by Equation (1.20). The irreps for the JT problems of interest are listed in Table 1.2. 
Table 1.2 The Jahn-Teller problems of interest [24]

\begin{tabular}{|c|c|c|}
\hline $\begin{array}{l}\text { Point } \\
\text { group, } G\end{array}$ & $\begin{array}{c}\text { Jahn-Teller problem: } \\
\Gamma \otimes \Lambda \text { or } \Gamma \otimes \sum \lambda_{k}\end{array}$ & Comments \\
\hline $\begin{array}{l}D_{4 h} \\
O_{h}\end{array}$ & $\begin{array}{c}E \otimes\left(b_{1 g}+b_{2 g}\right) \\
E \otimes e\end{array}$ & $\begin{array}{l}\text { square } \rightarrow \text { rhombus }\left(b_{1 g}\right) \text { or rectangle }\left(b_{2 g}\right) \\
\text { octahedron } \rightarrow \text { tetragonal bipyramid (TBP) }\end{array}$ \\
\hline$O_{h}$ & $T \otimes\left(e+t_{2}\right)$ & octahedron $\rightarrow \operatorname{TBP}(e)$ or trigonal antiprism $\left(t_{2}\right)$ \\
\hline$I_{h}$ & $T \otimes h$ & icosahedron $\rightarrow$ pentagonal antiprism \\
\hline$I_{h}$ & $G \otimes(g+h)$ & $\begin{array}{l}\text { icosahedron } \rightarrow \text { pentagonal or trigonal symmetry }(h) \\
\text { icosahedron } \rightarrow \text { tetrahedral symmetry }(g)\end{array}$ \\
\hline$I_{h}$ & $H \otimes(g+2 h)$ & $\begin{array}{l}\text { icosahedron } \rightarrow \text { pentagonal or trigonal symmetry }(h) \\
\text { icosahedron } \rightarrow \text { tetrahedral symmetry }(g)\end{array}$ \\
\hline
\end{tabular}

In general a top-rep for the Jahn-Teller problem $\Gamma \otimes \Lambda$ is a graph embedded in a space of the same dimension as $\Lambda$, i.e., a space of dimension $[d(d+1) / 2]-1$ in which $d$ is the dimension of $\Gamma$. However, if $\Lambda$ can be reduced to a sum of irreps $\lambda_{k}$ such that $\sum \lambda_{k}=\Lambda$, then the corresponding top-rep can be related to a set of complete graphs on $d_{k}-1$ vertices designated as $K_{d_{k}-1}$ [51]. Furthermore, a complete graph $K_{n}$ can be embedded into (n-1)-dimensional space to give an $n$-simplex. For example, a 2 -simplex is a $K_{3}$ graph (i.e., a triangle) embedded into 2-dimensional space (i.e., a plane). Similarly, a 3 -simplex is a $K_{4}$ graph (i.e., a tetrahedron) embedded into 3-dimensional space.

The point group symmetries for which JT distortions are of interest are the $D_{4 h}$ symmetry of the square, the $T_{d}$ symmetry of the tetrahedron, the $O_{h}$ symmetry of the cube or octahedron, and the $I_{h}$ symmetry of the icosahedron, dodecahedron, or $\mathrm{C}_{60}$. The important features of the top-reps of these JT distortions are discussed below.

\subsubsection{Square Planar Molecules}

JT distortions of the square are of interest from the chemical point of view in connection with distortions of cyclobutadiene derivatives from squares to rectangles (Figure 1.17a). In the $D_{4 h}$ symmetry of square planar molecules the only degenerate irreps are doubly degenerate $E$ representations. The corresponding product $\Lambda=[E \times E]_{\mathrm{S}}-A_{1}$ is a two-dimensional representation, which is reducible to a sum of the one-dimensional irreps $b_{1 g}$ and $b_{2 g}$ corresponding to the $E \otimes\left(b_{1 g}+b_{2 g}\right)$ problem. The corresponding top-rep consists of two crossed straight lines, which may be regarded as diagonals of a rhombus (dashed lines in Figure 1.17b). Note that a straight line plus its endpoints is a $K_{2}$ complete graph, which is the simplex in one-dimensional space.

One of the two diagonals in this top-rep for the $E \otimes\left(b_{1 g}+b_{2 g}\right)$ problem (the 'vertical' diagonal in Figure 1.17b) corresponds to the $b_{1 g}$ irrep with its endpoints corresponding to rhombus isomers of $D_{2 h}$ symmetry in which the two-fold axes are the diagonals of the rhombus. The enantiomer count 


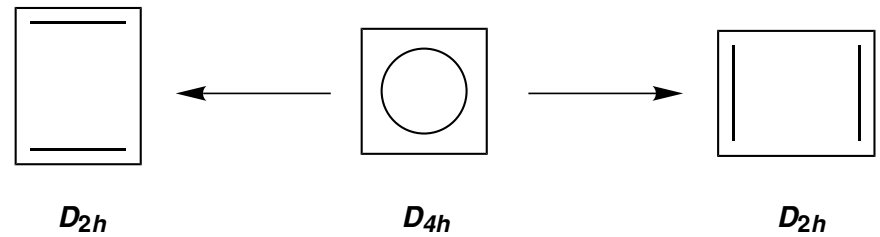

Cyclobutadiene

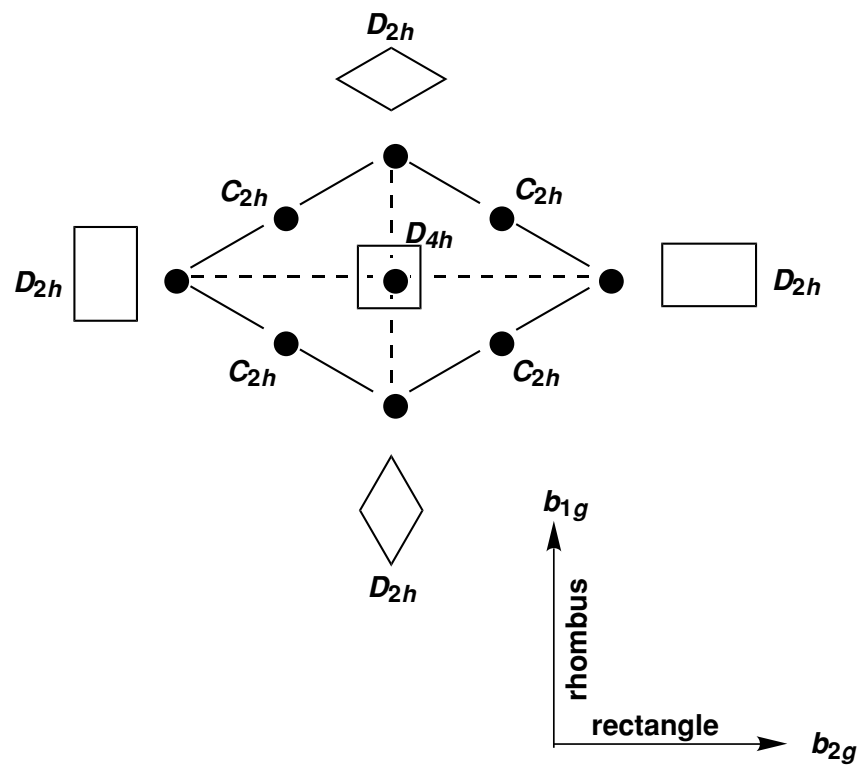

Figure 1.17 (a) The JT distortion of square $\left(D_{4 h}\right)$ to rectangular $\left(D_{2 h}\right)$ cyclobutadiene. (b) A rhombus as a top-rep for the $E \otimes\left(b_{1 g}+b_{2 g}\right)$ problem showing the distortions from a square $\left(D_{4 h}\right)$ to rectangles $\left(D_{2 h}\right)$ and rhombi $\left(D_{2 h}\right)$.

$E_{\text {rhomb }}=\left|D_{4 h}\right| /\left|D_{2 h}\right|=16 / 8=2$ by Equation (1.17) corresponds to the two endpoints of this diagonal. Similarly the other diagonal in this top-rep (the 'horizontal' diagonal in Figure 1.15b) corresponds to the $b_{2 g}$ irrep with its endpoints corresponding to the rectangle isomers, likewise of $D_{2 h}$ symmetry, but with the two-fold axes bisecting the midpoints of the rectangle edges. Again the enantiomer count $E_{\text {rect }}=\left|D_{4 h}\right| /\left|D_{2 h}\right|=16 / 8=2$ corresponds to the two endpoints of this diagonal. The distortion of square cyclobutadiene, $\mathrm{C}_{4} \mathrm{H}_{4}$, to the rectangular isomer (Figure 1.17a) corresponds to the latter diagonal $[88,89]$.

The diagonals in this top-rep of the $E \otimes\left(b_{1 g}+b_{2 g}\right)$ problem (Figure 1.17b) are drawn with different lengths corresponding to the non-equivalence (independence) of the $b_{1 g}$ and the $b_{2 g}$ components of this JT problem. Joining the 
end-points of these perpendicular diagonals of nonequal lengths generates a rhombus. The midpoints of the four edges of this rhombus correspond to isomers of $C_{2 h}$ kernel symmetry $[83,84]$ intermediate between the rectangle and rhombus joined by the edge in question. Note again that the enantiomer count of these edge midpoints $E_{C 2 h}=\left|D_{4 h}\right| /\left|C_{2 h}\right|=16 / 4=4$ corresponds to the four edges of the top-rep (Figure 1.17b).

\subsubsection{Octahedral Molecules}

JT distortions of octahedral molecules are of interest in coordination chemistry when the central atom (normally a d-block transition metal) has a partially filled $t_{2 g}$ or $e_{g}$ shell thereby leading to degenerate electronic states. The simplest JT distortion of an octahedron arises from electronic degeneracies in the molecular orbitals represented by the doubly degenerate $E$ representation. The corresponding product $\Lambda=[E \times E]_{\mathrm{S}}-A_{1}$ is the irreducible two-dimensional representation $e$, which leads to the $E \otimes e \mathrm{JT}$ problem. The corresponding top-rep is the 2-simplex, namely an equilateral triangle. In such a top-rep (Figure 1.18a) the vertices correspond to tetragonal bipyramidal $\left(D_{4 h}\right)$ isomers, which are either elongated or compressed along one of the original four-fold axes of the octahedron. Since there are three such four-fold axes, which are mutually perpendicular, there are necessarily three distinct tetragonal bipyramidal isomers corresponding not only to the three vertices of the triangle but also to the enantiomer count $E_{\text {tetrag }}=\left|O_{h}\right| /\left|D_{4 h}\right|=48 / 16=3$ by Equation (1.17). In fact these three vertices correspond to the three minima of the adiabatic potential surface considering both the linear and quadratic terms of the vibronic interaction $[23,24]$. The three edge midpoints correspond to orthorhombic isomers of $D_{2 h}$ kernel symmetry arising as intermediates in the conversion of one tetragonal bipyramidal isomer to another. Octahedral $\mathrm{d}^{9} \mathrm{Cu}$ (II) complexes such as $\mathrm{Cu}\left(\mathrm{H}_{2} \mathrm{O}\right)_{6}{ }^{2+}$ provide examples of $E \otimes e \mathrm{JT}$ distortions in which a pair of axial bonds of the octahedron is elongated to form a $D_{4 h}$ tetragonal bipyramid (Figure 1.18b) [90-92].

The other general type of JT distortion of octahedral molecules arises from electronic degeneracies in the molecular orbitals represented by the triply degenerate $T$ representation. In this case the corresponding product $\Lambda=[E \times E]_{\mathrm{S}}-A_{1}$ is a five-dimensional representation, which necessarily is reducible, since the octahedral point groups contain no five-dimensional irreps. Reducing this representation to a sum of irreps gives $e+t_{2}$ leading to the JT $T \otimes\left(e+t_{2}\right)$ problem. Analogy with the two-dimensional rhombus top-rep for the $E \otimes\left(b_{1 g}+b_{2 g}\right)$ problem (Figure 1.17b) discussed above suggests a fivedimensional top-rep with a tetrahedron for the $t_{2}$ component in three of the five dimensions and a triangle for the $e$ component in the other two dimensions of the five-dimensional space. However, the dimensionality of the top-rep can be reduced from five dimensions visualized only with considerable difficulty to a more readily visualized three-dimensional polyhedron if one type of distortion 

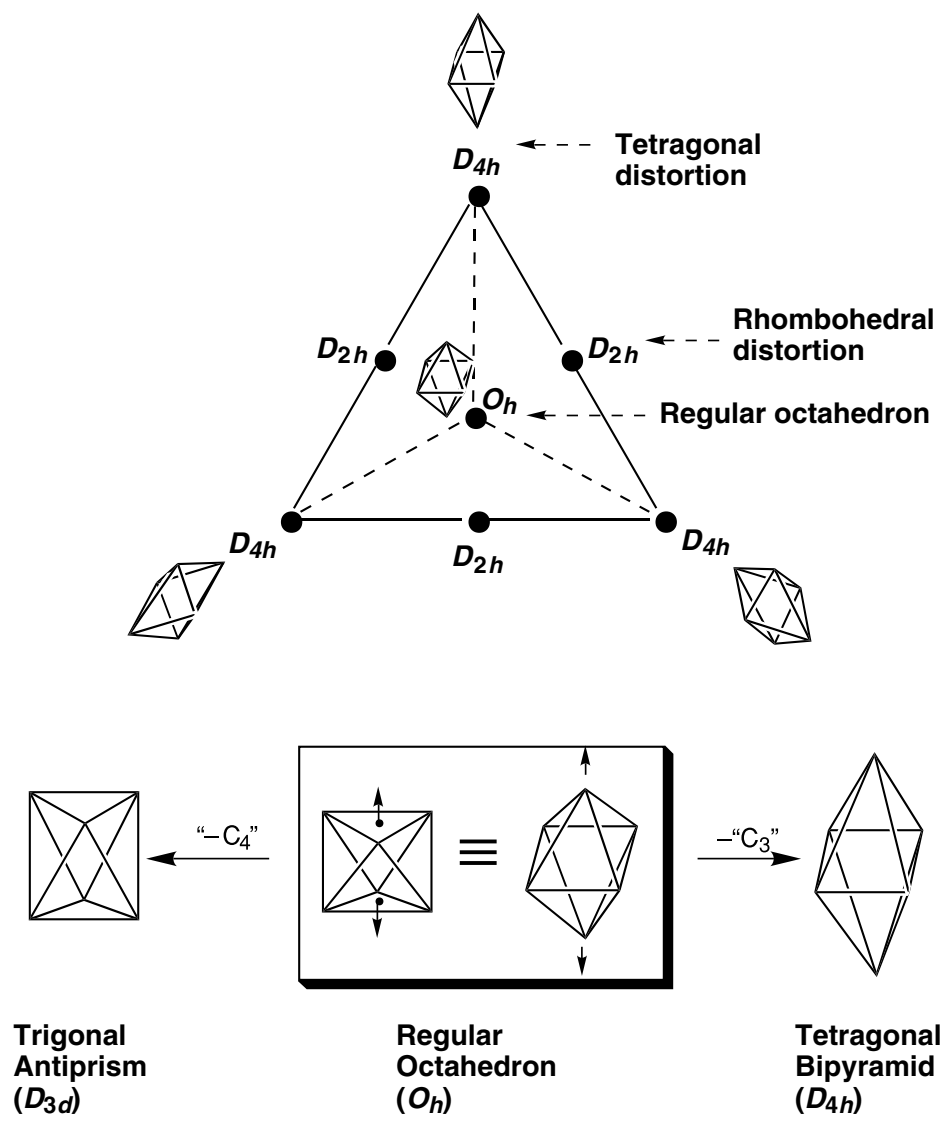

Figure 1.18 (a) Equilateral triangle top-rep for the $E \otimes e$ problem showing the distortions from a regular octahedron $\left(O_{h}\right)$ to the three elongated tetragonal bipyramidal isomers $\left(D_{4 h}\right)$. (b) The JT distortions of the regular octahedron to the elongated trigonal antiprism $\left(D_{3 d}\right)$ and elongated tetragonal bipyramid $\left(D_{4 h}\right)$.

corresponds to the vertices and the other type of distortion corresponds to the face midpoints of the polyhedron in question. The edges of the polyhedron are not the actual edges of the top-rep. However, their midpoints represent polyhedral isomers of lower symmetry than the isomers represented by either the vertices or the face midpoints. The edges belonging to the top-rep connect polyhedral edge midpoints to either vertices or face midpoints. The numbers of points in such a polyhedral top-rep corresponding to a distortion of the original point group $G$ (namely $O_{h}$ in this case) to a subgroup $H_{k}$ does not correspond to the enantiomer count $E=|G| /\left|H_{k}\right|$ (see Equation 1.17) but instead to the isomer count $I=|G| /\left|R_{k}\right|$ (Equation 1.16) where $R_{k}$ is the rotational 


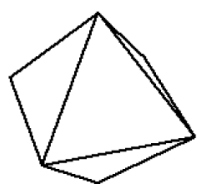

Octahedron

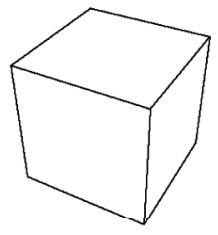

Cube

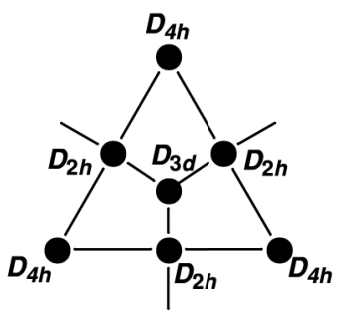

Octahedron face

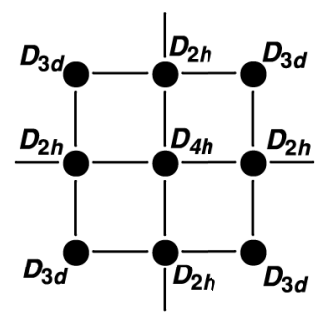

Cube face

Figure 1.19 (a) The regular octahedron as a top-rep for the $T \otimes\left(e+t_{2}\right)$ distortions of a regular octahedron showing on a sample face the locations of the tetragonal bipyramid isomers $\left(D_{4 h}\right)$, the trigonal antiprism isomers $\left(D_{3 d}\right)$, and the rhombohedral $D_{2 h}$ isomers as vertices, face midpoints, and edge midpoints, respectively. (b) The dual of the top-rep in Figure 1.19a based on a cube with the tetragonal bipyramid isomers $\left(D_{4 h}\right)$, the trigonal antiprism isomers $\left(D_{3 d}\right)$, and the rhombohedral $D_{2 h}$ isomers as face midpoints, vertices, and edge midpoints, respectively.

subgroup of $H_{k}$. For achiral $H_{k}$ (as in all of the examples discussed in this paper), $\left|H_{k}\right|=2\left|R_{k}\right|$ so that $I=2 E$.

These considerations lead to a top-rep for the $T \otimes\left(e+t_{2}\right)$ problem based on a regular octahedron (Figure 1.19a) with special points corresponding to the following isomers:

(1) The center of the octahedron corresponding to the symmetrical $O_{h}$ regular octahedron before any distortion.

(2) The six vertices of the octahedron corresponding to the three tetragonal bipyramidal $D_{4 h}$ isomers each considered as an enantiomeric pair $\left(I_{\text {tetrag }}=\right.$ $\left.\left|O_{h}\right| /\left|D_{4}\right|=48 / 8=6\right)$ corresponding to the $T \otimes e$ portion of the $T \otimes$ $\left(e+t_{2}\right)$ problem.

(3) The midpoints of the eight faces of the octahedron corresponding to the four trigonal antiprismatic $D_{3 d}$ isomers each considered as an enantiomeric pair $\left(I_{\text {trig }}=\left|O_{h}\right| /\left|D_{3}\right|=48 / 6=8\right)$ corresponding to the $T \otimes t_{2}$ portion of the $T \otimes\left(e+t_{2}\right)$ problem. 
(4) The midpoints of the 12 edges of the octahedron corresponding to the six orthorhombic $D_{2 h}$ isomer intermediates between either pairs of tetragonal bipyramids $\left(D_{4 h}\right)$ or pairs of trigonal antiprisms $\left(D_{3 d}\right)$ each considered as an enantiomeric pair $\left(I_{D 2 h}=\left|O_{h}\right| /\left|D_{2}\right|=48 / 4=12\right)$.

The dual of the octahedron, namely the cube, can also be used for a top-rep of the $T \otimes\left(e+t_{2}\right)$ problem in which the roles of the vertices and face midpoints are reversed from those of the octahedral top-rep. Thus for the cubic top-rep of the $T \otimes\left(e+t_{2}\right)$ problem (Figure 1.19b) the vertices represent the eight $D_{3 d}$ trigonal antiprismatic isomers and the face midpoints represent the six $D_{4 h}$ tetragonal bipyramid isomers. The edge midpoints of the cubic top-rep correspond to the $D_{2 h}$ orthorhombic intermediates as in the octahedral top-rep.

\subsubsection{Icosahedral Molecules}

JT distortions of icosahedral symmetry are of interest for understanding possible distortions in $I_{h}-\mathrm{C}_{60}$ [93] or $\mathrm{B}_{12} \mathrm{X}_{12}{ }^{2-}$ [94] upon reduction or oxidation. In this connection icosahedral molecules have $T, G$, and $H$ molecular orbitals of degeneracies 3,4 , and 5 , respectively, in which electronic degeneracies can occur. However, the non-symmetric parts of the symmetric direct products $\Lambda$ (Equation 1.20) for any of these degenerate irreps never contain any triply degenerate $t$ irreps (Table 1.2). For this reason the only possible JT distortions of icosahedra are those corresponding to the $g$ and $h$ irreps. The $g$ irrep corresponds to distortions reducing the symmetry from icosahedral to tetrahedral $\left(T_{h}\right)$. Similarly the $h$ irrep corresponds to distortions reducing the symmetry from icosahedral to either trigonal $\left(D_{3 d}\right)$ or pentagonal $\left(D_{5 d}\right)$. Reasonable topreps can be generated for both of these distortion pathways.

The simplest JT distortion of icosahedral molecules arises from electronic degeneracies in the molecular orbitals represented by the triply degenerate $T$ representation. The corresponding product $\Lambda=[T \times T]_{\mathrm{S}}-A_{1}$ is the fivedimensional irrep $h$, which leads to the $T \otimes h$ JT problem. The corresponding top-rep is the 5-simplex, which corresponds to the embedding of the complete graph on six vertices $\left(K_{6}\right)$ into five-dimensional space. This simplex has $(6 \times 5) /(2 \times 1)=15$ edges and $(6 \times 5 \times 4) /(3 \times 2 \times 1)=20$ two-dimensional faces, which themselves are necessarily 2 -simplices, i.e., triangles. Isomers are indicated by the following special points on this 5 -simplex:

(1) The center of the 5-simplex corresponding to the regular icosahedron $I_{h}$ before distortion.

(2) The six vertices of the 5-simplex corresponding to the six $D_{5 d}$ bicapped pentagonal antiprismatic isomers $\left(I_{D 5 d}=\left|I_{h}\right| /\left|D_{5 d}\right|=120 / 20=6\right)$.

(3) The midpoints of the 15 edges of the 5-simplex corresponding to the 15 $D_{2 h}$ intermediates $\left(I_{D 2 h}=\left|I_{h}\right| /\left|D_{2 h}\right|=120 / 8=15\right)$. 
(4) The midpoints of the 20 triangular faces of the 5-simplex corresponding to the $10 D_{3 d}$ trigonal isomers considered as enantiomeric pairs ( $E_{D 3 d}=$ $\left.\left|I_{h}\right| /\left|D_{3}\right|=120 / 6=20\right)$.

In order to avoid the complexities of five-dimensional space for a top-rep of the $T \otimes h$ problem, an alternative three-dimensional polyhedral representation can be generated based on the regular icosahedron (Figure 1.20a) analogous to the use of the regular octahedron for a top-rep of the related $T \otimes\left(e+t_{2}\right)$ problem. In such a representation, the special points on the icosahedron have the following significance:

(1) The center of the icosahedron corresponds to the symmetrical $I_{h}$ regular icosahedron before distortion.

(2) The 12 vertices of the icosahedron correspond to the six bicapped pentagonal antiprismatic $D_{5 d}$ isomers each considered as an enantiomeric pair $\left(I_{\text {pentag }}=\left|I_{h}\right| /\left|D_{5}\right|=120 / 10=12\right)$.

(3) The midpoints of the 20 faces of the icosahedron correspond to the 10 trigonal $D_{3 d}$ isomers each considered as an enantiomeric pair $\left(I_{\text {trig }}=\left|I_{h}\right| /\left|D_{3}\right|=\right.$ $120 / 6=20)$.

(4) The midpoints of the 30 edges of the icosahedron correspond to the 15 $D_{2 h}$ isomer intermediates between either pairs of pentagonal $D_{5 d}$ isomers or trigonal $D_{3 d}$ isomers each considered as an enantiomeric pair $\left(I_{D 2 h}=\right.$ $\left.\left|I_{h}\right| /\left|D_{2}\right|=120 / 4=30\right)$.

Alternatively, analogous to the $T \otimes\left(e+t_{2}\right)$ problem discussed above, the dual of the icosahedron, namely the regular dodecahedron, can be used for a toprep of the $T \otimes h$ problem (Figure 1.20b). In this case the roles of the vertices and face midpoints are reversed from those of the icosahedron top-rep (Figure 1.20a).

The next most complicated JT system of distortions for the regular icosahedron corresponds to the $G \otimes(g+h)$ problem. This problem generates a top-rep in nine-dimensional space combining a 4-simplex corresponding to the $g$ component (i.e., a $G \otimes g$ problem) with a 5-simplex corresponding to the $h$ component (i.e., a $G \otimes h$ problem). The $G \otimes h$ component involves distortions of $I_{h}$ symmetry to the lower $D_{5 d}, D_{3 d}$, and $D_{2 h}$ symmetries leading to top-reps similar to those discussed above for the related $T \otimes h$ problem.

The $G \otimes g$ component of the $G \otimes(g+h)$ problem leads to a new simplicial top-rep which is four-dimensional in accord with the dimension of the irrep $g$ for the corresponding distortion of the nuclear coordinates. This representation is based on the 4-simplex, which has five vertices and $(5 \times 4) /(2 \times 1)=10$ edges. This simplex is the four-dimensional analogue of the regular tetrahedron with a skeleton corresponding to the complete graph $K_{5}$ (Figure 1.21). In this top-rep the special points on the 4-simplex have the following significance: 


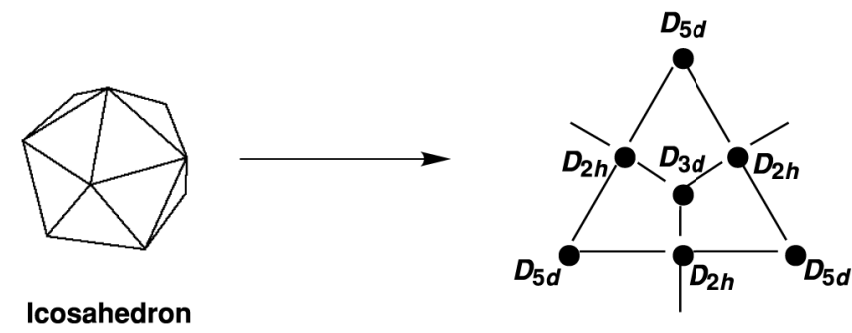

Icosahedron face

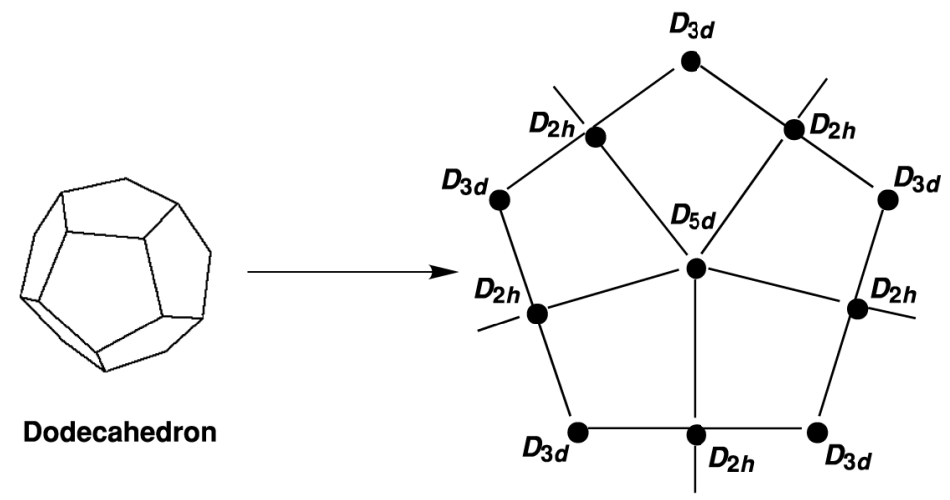

Dodecahedron face

Figure 1.20 (a) The regular icosahedron as a top-rep for the $T \otimes h$ distortions of a regular icosahedron showing on a sample face the locations of the $D_{5 d}, D_{3 d}$, and $D_{2 h}$ isomers as vertices, face midpoints, and edge midpoints, respectively. (b) The dual of the top-rep in Figure $1.20 \mathrm{a}$ based on a regular dodecahedron with $D_{5 d}, D_{3 d}$, and $D_{2 h}$ isomers as face midpoints, vertices, and edge midpoints, respectively.

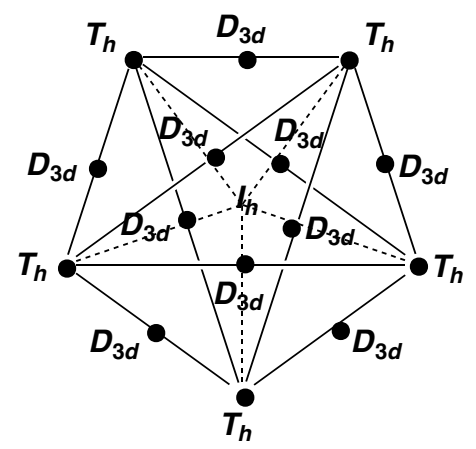

Figure 1.21 The $K_{5}$ skeleton of the 4-simplex (four-dimensional analogue of the regular tetrahedron) as a top-rep for the $G \otimes g$ distortions of an $\left(I_{h}\right)$ regular icosahedron (center point) to $T_{h}$ isomers (vertices) and $D_{3 d}$ intermediates (edge midpoints). 
(1) The center of the 4-simplex corresponds to the regular icosahedron $I_{h}$ before distortion.

(2) The five vertices of the 4-simplex correspond to the five $T_{h}$ isomers of tetrahedral symmetry $\left(E_{T h}=\left|I_{h}\right| /\left|T_{h}\right|=120 / 24=5\right)$.

(3) The midpoints of the 10 edges of the 4-simplex correspond to the ten $D_{3 d}$ intermediates in the interconversions of the $T_{h}$ isomers $\left(E_{D 3 d}=\left|I_{h}\right| /\left|D_{3 d}\right|=\right.$ $120 / 12=10)$.

The remaining JT system of distortions for the regular icosahedron corresponds to the $H \otimes(g+2 h)$ problem. A complete and rigorous top-rep of this system would require no less than 14 dimensions and is obviously impractical. The $g$ and $h$ components of this problem (i.e., the $H \otimes g$ and the $H \otimes h$ components) would have individual top-reps similar to those discussed above for the $G \otimes g$ and $T \otimes h$ problems, and introduce no fundamentally new features.

\section{ACKNOWLEDGMENT}

I am indebted to the National Science Foundation for partial support of this work under grant CHE-0209857.

\section{REFERENCES}

1. E. L. Muetterties, J. Am. Chem. Soc., 90, 5097 (1968).

2. E. L. Muetterties, J. Am. Chem. Soc., 91, 1636 (1969).

3. E. L. Muetterties, J. Am. Chem. Soc., 91, 4115 (1969).

4. E. L. Muetterties and A. T. Storr, J. Am. Chem. Soc., 91, 3098 (1969).

5. M. Gielen and J. Nasielski, Bull. Soc. Chim. Belges, 78, 339 (1969).

6. M. Gielen and J. Nasielski, Bull. Soc. Chim. Belges, 78, 351 (1969).

7. M. Gielen, C. Depasse-Delit, and J. Nasielski, Bull. Soc. Chim. Belges, 78, 357 (1969).

8. M. Gielen and C. Depasse-Delit, Theor. Chim. Acta, 14, 212 (1969).

9. M. Gielen, G. Mayence, and J. Topart, J. Organometal. Chem., 18, 1 (1969)

10. M. Gielen, M. de Clercq, and J. Nasielski, J. Organometal. Chem., 18, 217 (1969).

11. M. Gielen and N. Vanlautem, Bull. Soc. Chim. Belges, 79, 679 (1970).

12. M. Gielen, Bull. Soc. Chim. Belges, 80, 9 (1971).

13. J. I. Musher, J. Am. Chem. Soc., 94, 5662 (1972).

14. J. I. Musher, Inorg. Chem., 11, 2335 (1972).

15. W. G. Klemperer, J. Chem. Phys., 56, 5478 (1972).

16. W. G. Klemperer, J. Am. Chem. Soc., 94, 6940 (1972).

17. W. G. Klemperer, J. Am. Chem. Soc., 94, 8360 (1972).

18. J. Brocas, Top. Curr. Chem., 32, 43 (1972).

19. J. Brocas, in Advances in Dynamic Stereochemistry, edited by M. Gielen, Vol. 1, Freund, TelAviv, 1985, pp. 43-88.

20. R. B. King, Mol. Phys., 100, 1567 (2002).

21. I. B. Bersuker, Coord. Chem. Rev., 14, 357 (1975).

22. I. B. Bersuker, The Jahn-Teller Effect and Vibronic Interactions in Modern Chemistry, Plenum Press, New York, 1984. 
23. I. B. Bersuker, Electronic Structure and Properties of Transition Metal Compounds, Wiley, New York, 1996.

24. I. B. Bersuker, Chem. Rev., 101, 1067 (2001).

25. R. B. King in Advances in Dynamic Stereochemistry, edited by M. Gielen, Vol. 2, Freund Publishing Co., Tel Aviv, 1988, pp. 1-36.

26. R. B. King, in Graph Theoretical Approach to Chemical Reactivity, edited by D. Bonchev and O. Menkenyan, Kluwer, Dordrecht, The Netherlands, 1994, pp. 109135.

27. R. B. King, Applications of Graph Theory and Topology in Inorganic Cluster and Coordination Chemistry, CRC Press, Boca Raton, Florida, 1992, Chapter 12.

28. R. B. King, J. Am. Chem. Soc., 91, 7211 (1969).

29. F. Harary and E. M. Palmer, Graphical Enumeration, Academic Press, New York, 1973, p. 224.

30. W. T. Tutte, J. Combin. Theory Ser. B., 28, 105 (1980).

31. A. J. W. Duijvestijn and P. J. Federico, Math. Comput., 37, 523 (1981).

32. P. J. Federico, Geom. Ded., 3, 469 (1975).

33. D. Britton and J. D. Dunitz, Acta Crystallegr., A29, 362 (1973).

34. R. B. King, Theor. Chim. Acta, 64, 453 (1984).

35. R. B. King, Inorg. Chem., 37, 3057 (1998).

36. B. Grünbaum, Convex Polytopes, Interscience, New York, 1967.

37. F. A. Cotton, Acc. Chem. Res., 1, 257 (1968).

38. W. N. Lipscomb, Science, 153, 373 (1966).

39. R. S. Berry, J. Chem. Phys., 32, 933 (1960).

40. R. B. King, Inorg. Chim. Acta, 49, 237 (1981).

41. B. M. Gimarc and J. J. Ott, Inorg. Chem., 25, 83 (1986).

42. J. J. Ott, C. A. Brown, and B. M. Gimarc, Inorg. Chem., 28, 4269 (1989).

43. B. M. Gimarc and J. J. Ott, Inorg. Chem., 25, 2708 (1986).

44. R. R. Holmes, Accts. Chem. Res., 5, 296 (1972).

45. L. J. Guggenberger and E. L. Muetterties, J. Am. Chem. Soc., 98, 7221 (1976).

46. D. J. Wales and A. J. Stone, Inorg. Chem., 26, 3845 (1987).

47. D. M. P. Mingos and R. J. Johnston, Polyhedron, 7, 2437 (1988).

48. D. J. Wales, D. M. P. Mingos, and L. Zhenyang, Inorg. Chem., 28, 2754 (1989).

49. A. Ceulemans, G. Goijens, and M. T. Nguyen, J. Am. Chem. Soc., 116, 9395 (1994).

50. R. B. King, J. Mol. Struct. THEOCHEM, 185, 15 (1989).

51. L. W. Beinecke and R. J. Wilson, Selected Topics in Graph Theory, Academic Press, New York, 1978.

52. P. McMullen and G. C. Shephard, Convex Polytopes and the Upper Bound Conjecture, Cambridge University Press, Cambridge, England, 1971.

53. P. McMullen and G. C. Shephard, Mathematika, 15, 223 (1968).

54. R. B. King, MATCH-Commun. Math. Chem., 48, 155 (2003).

55. J. C. Bailar, Jr., J. Inorg. Nucl. Chem., 8, 165 (1985).

56. P. Ray and N. K. Dutt, J. Indian Chem. Soc., 20, 81 (1943).

57. R. B. King and A. J. W. Duijvestijn, Inorg. Chim. Acta, 178, 55 (1990).

58. J. Bould, J. D. Kennedy, and M. Thornton-Pett, J. Chem. Soc. Dalton, 563 (1992).

59. J. D. Kennedy and B. Štibr, in Current Topics in the Chemistry of Boron, edited by G. W. Kabalka, Royal Society of Chemistry, Cambridge, UK, 1994, pp. 285-292.

60. J. D. Kennedy, in The Borane-Carborane-Carbocation Continuum, edited by J. Casanova, Wiley, New York, 1998, Chapter 3, pp. 85-116.

61. B. Štibr, J. D. Kennedy, E. Drdáková, and M. Thornton-Pett, J. Chem. Soc. Dalton Trans., 229 (1994).

62. R. B. King, Inorg. Chem., 35, 5151 (1999).

63. R. B. King, Inorg. Chim. Acta, 300-302, 537 (2000). 
64. J. Bould, J. E. Crook, N. N. Greenwood, J. D. Kennedy, and W. S. McDonald, Chem. Commun., 346 (1982).

65. R. B. King, Inorg. Chem., 40, 2699 (2001).

66. A. T. Balaban, D. Farcaşiu, and R. Banica, Rev. Roum. Chim., 11, 1205 (1966).

67. B. M. Gimarc and J. J. Ott, in Graph Theory and Topology in Chemistry, edited by R. B. King and D. H. Rouvray, Elsevier, Amsterdam, 1978, pp. 285-301.

68. F. J. Budden, The Fascination of Groups, Cambridge University Press, London, 1972.

69. C. D. H. Chisholm, Group Theoretical Techniques in Quantum Chemistry, Academic Press, New York, 1976.

70. J. A. Pople, J. Am. Chem. Soc., 102, 4615 (1980).

71. S. Fujita, Symmetry and Combinatorial Enumeration in Chemistry, Springer, Berlin, 1991.

72. M. Randić, Croat. Chim. Acta, 74, 683 (2001).

73. M. Baudler, Angew. Chem. Int. Ed. Engl., 21, 492 (1982).

74. G. Pólya, Acta Math., 68, 143 (1937).

75. N. Debruijn in Applied Combinatorial Mathematics, edited by E. F. Beckenbach, Wiley, New York, 1964.

76. J. G. Nourse and K. Mislow, J. Am. Chem. Soc., 97, 4571 (1975).

77. R. B. King, Inorg. Chem., 20, 363 (1981).

78. R. B. King, Theor. Chim. Acta, 59, 25 (1981).

79. N. L. Biggs, Algebraic Graph Theory, Cambridge University Press, London, 1974.

80. B. Grünbaum, Convex Polytopes, Interscience, New York, 1967.

81. H. S. M. Coxeter, Regular Polytopes, MacMillan, New York, Second Edition, 1963.

82. H. S. M. Coxeter, Regular Complex Polytopes, Cambridge University Press, Cambridge, UK, Second Edition, 1991.

83. A. Ceulemans, D. Beyens, and L. G. Vanquickenborne, J. Am. Chem. Soc., 106, 5824 (1984).

84. A. Ceulemans and L. G. Vanquickenborne, Structure and Bonding, 71, 125 (1989).

85. M. Orchin and H. H. Jaffé, Symmetry, Orbitals, and Spectra, Wiley, New York, 1971, pp. 192-194.

86. R. L. Ellis and H. H. Jaffé, J. Chem. Educ., 48, 92 (1971).

87. H. A. Jahn and E. Teller, Proc. Soc. London Ser. A, 161, 220 (1937).

88. V. I. Minkin, M. N. Glukhovtsev, and B. Ya. Simkin, Aromaticity and Antiaromaticity: Electronic and Structural Aspects, Wiley-Interscience, New York, 1994.

89. K. B. Wiberg, Chem. Rev., 101, 1317 (2001).

90. B. Bleany and K. D. Bowers, Proc. Phys. Soc. (London), A65, 667 (1952).

91. R. Engelman, The Jahn-Teller Effect in Molecules and Crystals, Wiley-Interscience, New York, 1972.

92. K. F. Purcell and J. C. Kotz, An Introduction to Inorganic Chemistry, Saunders, Philadelphia, PA, 1980.

93. C. C. Chancey and M. C. M. O'Brien, The Jahn-Teller Effect in $C_{60}$ and Other Icosahedral Molecules, Princeton University Press, Princeton, N. J., 1997.

94. T. Peymann, C. B. Knobler, S. I. Khan, and M. F. Hawthorne, Angew. Chem. Int. Ed. Engl., 40, 1664 (2001). 
